\title{
Uniform Convergence and Spectra of Operators in a Class of Fréchet Spaces
}

\author{
Angela A. Albanese, ${ }^{1}$ José Bonet, $^{2}$ and Werner J. Ricker ${ }^{3}$ \\ ${ }^{1}$ Dipartimento di Matematica e Fisica "E. De Giorgi”, Università del Salento, C.P.193, 73100 Lecce, Italy \\ ${ }^{2}$ Instituto Universitario de Matemática Pura y Aplicada IUMPA, Universitat Politècnica de València, 46071 Valencia, Spain \\ ${ }^{3}$ Math.-Geogr. Fakultät, Katholische Universität Eichstätt-Ingolstadt, 85072 Eichstätt, Germany
}

Correspondence should be addressed to José Bonet; jbonet@mat.upv.es

Received 22 October 2013; Accepted 20 December 2013; Published 30 January 2014

Academic Editor: Alfredo Peris

Copyright (C) 2014 Angela A. Albanese et al. This is an open access article distributed under the Creative Commons Attribution License, which permits unrestricted use, distribution, and reproduction in any medium, provided the original work is properly cited.

Well-known Banach space results (e.g., due to J. Koliha and Y. Katznelson/L. Tzafriri), which relate conditions on the spectrum of a bounded operator $T$ to the operator norm convergence of certain sequences of operators generated by $T$, are extended to the class of quojection Fréchet spaces. These results are then applied to establish various mean ergodic theorems for continuous operators acting in such Fréchet spaces and which belong to certain operator ideals, for example, compact, weakly compact, and Montel.

\section{Introduction}

Given a Banach space $X$ and a continuous linear operator $T$ on $X$, there are various classical results which relate conditions on the spectrum $\sigma(T)$ of $T$ with the operator norm convergence of certain sequences of operators generated by $T$. For instance, if $\lim _{n \rightarrow \infty}\left(\left\|T^{n}\right\|_{\text {op }} / n\right)=0$, with \|\|$_{\text {op }}$ denoting the operator norm, (even $T^{n} / n \rightarrow 0$ in the weak operator topology suffices), then necessarily $\sigma(T) \subseteq \overline{\mathbb{D}}$, where $\mathbb{D}:=$ $\{z \in \mathbb{C}:|z|<1\}$, [1, p. 709, Lemma 1]. The stronger condition $\lim _{n \rightarrow \infty}\left\|T^{n}\right\|_{\text {op }}=0$ is equivalent to the requirement that both $\sigma(T) \subseteq \mathbb{D}$ and $\lim _{n \rightarrow \infty}\left(\left\|T^{n}\right\|_{\text {op }} / n\right)=0$ hold [2]. An alternate condition, namely, that $\left\{T^{n}\right\}_{n=1}^{\infty}$ is a convergent sequence relative to the operator norm, is equivalent to the requirement that the three conditions $\lim _{n \rightarrow \infty}\left(\left\|T^{n}\right\|_{\text {op }} / n\right)=0$, the range $(I-T)^{m}(X)$ is closed in $X$ for some $m \in \mathbb{N}$, and $\Gamma(T) \subseteq\{1\}$ are satisfied [3]. Here $\Gamma(T):=\sigma(T) \cap \mathbb{T}$ with $\mathbb{\mathbb { T }}:=\{z \in \mathbb{C}:|z|=1\}$ being the boundary of $\mathbb{D}$. Such results as above are often related to the uniform mean ergodicity of $T$, meaning that the sequence of averages $\left\{(1 / n) \sum_{m=1}^{n} T^{m}\right\}$ of $T$ is operator norm convergent. For instance, if $\lim _{n \rightarrow \infty}\left(\left\|T^{n}\right\|_{o p} / n\right)=0$ and $1 \notin$ $\sigma(T)$, then $T$ is uniformly mean ergodic [4, p. 90, Theorem 2.7]. Or if $\lim _{n \rightarrow \infty}\left(\left\|T^{n}\right\|_{\text {op }} / n\right)=0$, then $T$ is uniformly mean ergodic if and only if $(I-T)(X)$ is closed [5].
Our first aim is to extend results of the above kind to the class of all Fréchet spaces referred to as prequojections; this is achieved in Section 3. The extension to the class of all Fréchet spaces is not possible; see Proposition 17 below and [6, Example 3.11], for instance. We point out that a classical result of Katznelson and Tzafriri stating, for any Banachspace-operator $T$ satisfying $\sup _{n \in \mathbb{N}}\left\|T^{n}\right\|_{\text {op }}<\infty$, that $\lim _{n \rightarrow \infty}\left\|T^{n+1}-T^{n}\right\|_{\text {op }}=0$ if and only if $\Gamma(T) \subseteq\{1\}$ [7], is also extended to prequojection Fréchet spaces; see Theorem 20.

Our second aim is inspired by well-known applications of the above mentioned Banach space results to determine the uniform mean ergodicity of operators $T$ which satisfy $\lim _{n \rightarrow \infty}\left(\left\|T^{n}\right\|_{\text {op }} / n\right)=0$ and belong to certain operator ideals, such as the compact or weakly compact operators; see, for example, [1, Ch. VIII, $\$ 8],[4$, Ch. $2, \S 2.2]$, and [8, Theorem 6.1], where $T$ can even be quasi-compact. An extension of such a mean ergodic result to the class of quasi-precompact operators acting in various locally convex Hausdorff spaces is presented in [9]. For prequojection Fréchet spaces, this result is further extended to the (genuinely) larger class of quasi-Montel operators; see Proposition 32, Remark 33, and Theorem 35. A mean ergodic theorem for Cesàro bounded, weakly compact operators (and also reflexive operators) in a certain class of locally convex spaces (which includes all 
Fréchet spaces), is also presented; see Proposition 23 and Remark 24(ii).

\section{Preliminaries and Spectra of Operators}

Let $X$ be a lcHs and $\Gamma_{X}$ a system of continuous seminorms determining the topology of $X$. The strong operator topology $\tau_{s}$ in the space $\mathscr{L}(X)$ of all continuous linear operators from $X$ into itself (from $X$ into another lcHs $Y$ we write $\mathscr{L}(X, Y)$ ) is determined by the family of seminorms $q_{x}(S):=q(S x)$, for $S \in \mathscr{L}(X)$, for each $x \in X$ and $q \in \Gamma_{X}$, in which case we write $\mathscr{L}_{s}(X)$. Denote by $\mathscr{B}(X)$ the collection of all bounded subsets of $X$. The topology $\tau_{b}$ of uniform convergence on bounded sets is defined in $\mathscr{L}(X)$ via the seminorms $q_{B}(S):=$ $\sup _{x \in B} q(S x)$, for $S \in \mathscr{L}(X)$, for each $B \in \mathscr{B}(X)$ and $q \in \Gamma_{X}$; in this case we write $\mathscr{L}_{b}(X)$. For $X$ a Banach space, $\tau_{b}$ is the operator norm topology in $\mathscr{L}(X)$. If $\Gamma_{X}$ is countable and $X$ is complete, then $X$ is called a Fréchet space. The identity operator on a lcHs $X$ is denoted by $I$.

By $X_{\sigma}$ we denote $X$ equipped with its weak topology $\sigma\left(X, X^{\prime}\right)$, where $X^{\prime}$ is the topological dual space of $X$. The strong topology in $X$ (resp. $X^{\prime}$ ) is denoted by $\beta\left(X, X^{\prime}\right)$ (resp. $\left.\beta\left(X^{\prime}, X\right)\right)$ and we write $X_{\beta}$ (resp. $X_{\beta}^{\prime}$ ); see $[10$, IV, Ch. 23] for the definition. The strong dual space $\left(X_{\beta}^{\prime}\right)_{\beta}^{\prime}$ of $X_{\beta}^{\prime}$ is denoted simply by $X^{\prime \prime}$. By $X_{\sigma}^{\prime}$ we denote $X^{\prime}$ equipped with its weak-star topology $\sigma\left(X^{\prime}, X\right)$. Given $T \in \mathscr{L}(X)$, its dual operator $T^{\prime}: X^{\prime} \rightarrow X^{\prime}$ is defined by $\left\langle x, T^{\prime} x^{\prime}\right\rangle=\left\langle T x, x^{\prime}\right\rangle$ for all $x \in X, x^{\prime} \in X^{\prime}$. It is known that $T^{\prime} \in \mathscr{L}\left(X_{\sigma}^{\prime}\right)$ and $T^{\prime} \in \mathscr{L}\left(X_{\beta}^{\prime}\right),[11$, p. 134].

For a Fréchet space $X$ and $T \in \mathscr{L}(X)$, the resolvent set $\rho(T)$ of $T$ consists of all $\lambda \in \mathbb{C}$ such that $R(\lambda, T):=(\lambda I-T)^{-1}$ exists in $\mathscr{L}(X)$. Then $\sigma(T):=\mathbb{C} \backslash \rho(T)$ is called the spectrum of $T$. The point spectrum $\sigma_{p}(T)$ consists of all $\lambda \in \mathbb{C}$ such that $(\lambda I-T)$ is not injective. Unlike for Banach spaces, it may happen that $\rho(T)=\emptyset$. For example, let $\omega=\mathbb{C}^{\mathbb{N}}$ be the Fréchet space equipped with the lc-topology determined via the seminorms $\left\{q_{n}\right\}_{n=1}^{\infty}$, where $q_{n}(x):=\max _{1 \leq j \leq n}\left|x_{j}\right|$, for $x=$ $\left(x_{j}\right)_{j=1}^{\infty} \in \omega$. Then the unit left shift operator $T: x \mapsto\left(x_{2}, x_{3}\right.$, $\left.x_{4}, \ldots\right)$, for $x \in \omega$, belongs to $\mathscr{L}(\omega)$ and, for every $\lambda \in \mathbb{C}$, the element $\left(1, \lambda, \lambda^{2}, \lambda^{3}, \ldots\right) \in \omega$ is an eigenvector corresponding to $\lambda$.

For a Fréchet space $X$, the natural imbedding $\Phi: X \rightarrow$ $X^{\prime \prime}$ is an isomorphism of $X$ onto the closed subspace $\Phi(X)$ of $X^{\prime \prime}$. Moreover, we always have

$$
S^{\prime \prime} \circ \Phi=\Phi \circ S, \quad S \in \mathscr{L}(X) ;
$$

that is, $S^{\prime \prime}$ is an extension of $S$.

The following result will be required in the sequel. Since the proof is standard we omit it. The polar of a set $\mathscr{U} \subseteq X$ is denoted by $\mathcal{U}^{\circ} \subseteq X^{\prime}$.

Lemma 1. Let $X$ be a Fréchet space.

(i) Let $\left\{p_{j}\right\}_{j=1}^{\infty} \subseteq \Gamma_{X}^{\prime \prime}$ be a fundamental, increasing sequence which determines the lc-topology of $X^{\prime \prime}$. For each $j \in \mathbb{N}$ define $q_{j}$ on $X$ via $q_{j}:=p_{j} \circ \Phi$. Then $\left\{q_{j}\right\}_{j=1}^{\infty} \subseteq \Gamma_{X}$ is a fundamental, increasing sequence which determines the lc-topology of $X$. (ii) Let $\left\{r_{j}\right\}_{j=1}^{\infty} \subseteq \Gamma_{X}$ be a fundamental, increasing sequence which determines the lc-topology of $X$. For each $j \in \mathbb{N}$, let $r_{j}^{\prime \prime}$ denote the Minkowski functional (in $\left.X^{\prime \prime}\right)$ of the bipolar of $\mathcal{U}_{j}:=r_{j}^{-1}([0,1]) \subseteq X$. Then $\left\{r_{j}^{\prime \prime}\right\}_{j=1}^{\infty} \subseteq \Gamma_{X}^{\prime \prime}$ is a fundamental, increasing sequence which determines the lc-topology of $X^{\prime \prime}$. Moreover, for each $j \in \mathbb{N}$, we have

$$
r_{j}(x)=\sup _{x^{\prime} \in \mathscr{U}_{j}^{\circ}}\left|\left\langle x, x^{\prime}\right\rangle\right|, \quad r_{j}^{\prime \prime}\left(x^{\prime \prime}\right)=\sup _{x^{\prime} \in \mathcal{U}_{j}^{\circ}}\left|\left\langle x^{\prime \prime}, x^{\prime}\right\rangle\right|
$$

for each $x \in X$ and $x^{\prime \prime} \in X^{\prime \prime}$. In particular, $r_{j}^{\prime \prime} \circ \Phi=r_{j}$; that is, the restriction of $r_{j}^{\prime \prime}$ to $X \simeq \Phi(X)$ coincides with $r_{j}$, for each $j \in \mathbb{N}$.

For Banach spaces the following fact is well-known.

Lemma 2. Let $X$ be a lcHs and $\left\{T_{n}\right\}_{n=1}^{\infty} \subseteq \mathscr{L}(X)$ be an equicontinuous sequence. Then also $\left\{T_{n}^{\prime \prime}\right\}_{n=1}^{\infty} \subseteq \mathscr{L}\left(X^{\prime \prime}\right)$ is equicontinuous.

Proof. Let $B \in \mathscr{B}(X)$. Then $C:=\cup_{n=1}^{\infty} T_{n}(B) \in \mathscr{B}(X)$ as $\left\{T_{n}\right\}_{n=1}^{\infty}$ is equicontinuous. So, for all $x^{\prime} \in X^{\prime}$ and $n \in \mathbb{N}$, we have $T_{n}^{\prime} x^{\prime} \in X_{\beta}^{\prime}$ with

$$
\begin{aligned}
p_{B}\left(T_{n}^{\prime} x^{\prime}\right) & :=\sup _{x \in B}\left|\left\langle x, T_{n}^{\prime} x^{\prime}\right\rangle\right| \\
& =\sup _{x \in B}\left|\left\langle T_{n} x, x^{\prime}\right\rangle\right| \leq \sup _{y \in C}\left|\left\langle y, x^{\prime}\right\rangle\right|=p_{C}\left(x^{\prime}\right) .
\end{aligned}
$$

As the seminorms $\left\{p_{B}: B \in \mathscr{B}(X)\right\}$ generate the lc-topology of $X_{\beta}^{\prime}$, the previous inequality shows that $\left\{T_{n}^{\prime}\right\}_{n=1}^{\infty} \subseteq \mathscr{L}\left(X_{\beta}^{\prime}\right)$ is equicontinuous.

Since $\left\{T_{n}^{\prime}\right\}_{n=1}^{\infty} \subseteq \mathscr{L}\left(X_{\beta}^{\prime}\right)$ is equicontinuous and the lctopology of $X^{\prime \prime}$ is generated by the polars of bounded subsets of $X_{\beta}^{\prime}$, the same argument as above yields that $\left\{T_{n}^{\prime \prime}\right\}_{n=1}^{\infty} \subseteq$ $\mathscr{L}\left(X^{\prime \prime}\right)$ is equicontinuous.

Lemma 3. Let $X$ be a Fréchet space and $T \in \mathscr{L}(X)$. Then $T$ is an isomorphism of $X$ onto itself if and only if $T^{\prime \prime}$ is an isomorphism of $X^{\prime \prime}$ onto itself.

Proof. If $T$ is an isomorphism of $X$ onto itself, then there exists $T^{-1} \in \mathscr{L}(X)$ with $T T^{-1}=T^{-1} T=I$. It follows that $T^{\prime}$, $\left(T^{-1}\right)^{\prime} \in \mathscr{L}\left(X_{\beta}^{\prime}\right)$ and so $T^{\prime \prime},\left(T^{-1}\right)^{\prime \prime} \in \mathscr{L}\left(X^{\prime \prime}\right)$. Accordingly, $I=\left(T T^{-1}\right)^{\prime \prime}=T^{\prime \prime}\left(T^{-1}\right)^{\prime \prime}$ and $I=\left(T^{-1} T\right)^{\prime \prime}=\left(T^{-1}\right)^{\prime \prime} T^{\prime \prime}$. Thus, $\left(T^{\prime \prime}\right)^{-1}$ exists in $\mathscr{L}\left(X^{\prime \prime}\right)$ and $\left(T^{\prime \prime}\right)^{-1}=\left(T^{-1}\right)^{\prime \prime}$; that is, $T^{\prime \prime}$ is an isomorphism of $X^{\prime \prime}$ onto itself.

Conversely, suppose that $T^{\prime \prime}$ is an isomorphism of $X^{\prime \prime}$ onto itself. Since $T^{\prime \prime}$ is an extension of $T$ (i.e., $T=\left.T^{\prime \prime}\right|_{X}$ ), we see that $T$ is one-to-one. Moreover, since $X$ is a closed subspace of $X^{\prime \prime}$ (as $X$ is a complete, barrelled lcHs), it follows that $T(X)=T^{\prime \prime}(X)$ is closed. It remains to show that $T(X)=$ $X$. But, if $T(X) \neq X$, then there is $f \in X^{\prime} \backslash\{0\}$ such that $\langle T x, f\rangle=\left\langle x, T^{\prime} f\right\rangle=0$ for all $x \in X$. Hence, $T^{\prime} f=0$; this is a contradiction because the surjectivity of $T^{\prime \prime}$ implies that $T^{\prime}$ is necessarily one-to-one. 
We remark that Lemma 3 remains valid for $X$ a complete barrelled lcHs.

The next result is an immediate consequence of (1) and Lemma 3.

Corollary 4. Let $X$ be a Fréchet space and $T \in \mathscr{L}(X)$. Then $\rho(T)=\rho\left(T^{\prime \prime}\right)$ and $\sigma(T)=\sigma\left(T^{\prime \prime}\right)$. Moreover,

$$
\Phi \circ R(\lambda, T)=R\left(\lambda, T^{\prime \prime}\right) \circ \Phi, \quad \lambda \in \rho(T)=\rho\left(T^{\prime \prime}\right) ;
$$

that is, the restriction of $R\left(\lambda, T^{\prime \prime}\right)$ to the closed subspace $X \simeq$ $\Phi(X)$ of $X^{\prime \prime}$ coincides with $R(\lambda, T)$. Briefly, $\left.R\left(\lambda, T^{\prime \prime}\right)\right|_{X}=$ $R(\lambda, T)$.

A Fréchet space $X$ is always a projective limit of continuous linear operators $R_{k}: X_{k+1} \rightarrow X_{k}$, for $k \in \mathbb{N}$, with each $X_{k}$ a Banach space. If $X_{k}$ and $R_{k}$ can be chosen such that each $R_{k}$ is surjective and $X$ is isomorphic to the projective limit $\operatorname{proj}_{j}\left(X_{j}, R_{j}\right)$, then $X$ is called a quojection [12, Section 5]. Banach spaces and countable products of Banach spaces are quojections. Actually, every quojection is the quotient of a countable product of Banach spaces [13]. In [14] Moscatelli gave the first examples of quojections which are not isomorphic to countable products of Banach spaces. Concrete examples of quojection Fréchet spaces are $\omega=$ $\mathbb{C}^{\mathbb{N}}$, the spaces $L_{\mathrm{loc}}^{p}(\Omega)$, with $1 \leq p \leq \infty$, and $C^{(m)}(\Omega)$ for $m \in \mathbb{N}_{0}$, with $\Omega \subseteq \mathbb{R}^{N}$ any open set, all of which are isomorphic to countable products of Banach spaces. The spaces of continuous functions $C(\Lambda)$, with $\Lambda$ a $\sigma$-compact, completely regular topological space, endowed with the compact open topology, are also quojections. Domański exhibited a completely regular topological space $\Lambda$ such that the Fréchet space $C(\Lambda)$ is a quojection which is not isomorphic to a complemented subspace of a product of Banach spaces, $[15$, Theorem]. A Fréchet space $X$ admits a continuous norm if and only if $X$ contains no isomorphic copy of $\omega[16$, Theorem 7.2.7]. On the other hand, a quojection $X$ admits a continuous norm if and only if it is a Banach space [12, Proposition 3]. So, a quojection is either a Banach space or contains an isomorphic copy of $\omega$, necessarily complemented, [16, Theorem 7.2.7]. Also [17] is relevant.

Lemma 5. Let $X$ be a quojection Fréchet space and $S \subseteq \mathscr{L}(X)$. Suppose that $X=\operatorname{proj}_{j}\left(X_{j}, Q_{j, j+1}\right)$, with $X_{j}$ a Banach space (having norm \|\|$_{j}$ ) and linking maps $Q_{j, j+1} \in \mathscr{L}\left(X_{j+1}, X_{j}\right)$ which are surjective for all $j \in \mathbb{N}$, and suppose, for each $j \in \mathbb{N}$, that there exists $S_{j} \in \mathscr{L}\left(X_{j}\right)$ satisfying

$$
S_{j} Q_{j}=Q_{j} S,
$$

where $Q_{j} \in \mathscr{L}\left(X, X_{j}\right), j \in \mathbb{N}$, denotes the canonical projection of $X$ onto $X_{j}$ (i.e., $Q_{j, j+1} \circ Q_{j+1}=Q_{j}$ ). Then

$$
\sigma(S) \subseteq \bigcup_{j=1}^{\infty} \sigma\left(S_{j}\right) \subseteq \sigma(S) \cup \bigcup_{j=1}^{\infty} \sigma_{p}\left(S_{j}\right) .
$$

Moreover,

$$
\sigma_{p}(S) \subseteq \bigcup_{j=1}^{\infty} \sigma_{p}\left(S_{j}\right)
$$

If, in addition, for every $\lambda \in \rho(S)$, the resolvent operator $R(\lambda, S)$ satisfies

$$
R(\lambda, S)\left(\operatorname{Ker} Q_{j}\right) \subseteq \operatorname{Ker} Q_{j}, \quad j \in \mathbb{N},
$$

then $\sigma(S)=\cup_{j=1}^{\infty} \sigma\left(S_{j}\right)$.

Proof. For the containments (6) and (7) we refer to [18, Lemma 5.1].

Suppose now that (8) holds for each $\lambda \in \rho(S)$. To establish the desired equality, let $\lambda \in \rho(S)$. Then $\lambda I-S$ is surjective. Fix $j \in \mathbb{N}$. Since $Q_{j}: X \rightarrow X_{j}$ is surjective, it is routine to check from the identity $\left(\lambda I_{j}-S_{j}\right) Q_{j}=Q_{j}(\lambda I-S)$ that also $\lambda I_{j}-S_{j}$ is surjective (with $I_{j} \in \mathscr{L}\left(X_{j}\right)$ the identity operator). To verify $\lambda I_{j}-S_{j}$ is injective suppose that $\left(\lambda I_{j}-S_{j}\right) y=0$ for some $y \in X_{j}$, in which case $y=Q_{j} x$ for some $x \in X$. Accordingly,

$$
Q_{j}(\lambda I-S) x=\left(\lambda I_{j}-S_{j}\right) Q_{j} x=\left(\lambda I_{j}-S_{j}\right) y=0
$$

shows that $(\lambda I-S) x \in \operatorname{Ker} Q_{j}$. It then follows from (8) that $x=R(\lambda, S)(\lambda I-S) x \in \operatorname{Ker} Q_{j}$; that is, $Q_{j} x=0$. Since $y=Q_{j} x$, we have $y=0$. Hence, $\lambda I_{j}-S_{j}$ is injective. This establishes that $\lambda \in \rho\left(S_{j}\right)$. Accordingly, $\rho(S)=\cap_{j=1}^{\infty} \rho\left(S_{j}\right)$ as desired.

The following result occurs in [18, Lemma 5.2].

Lemma 6. Let $X$ be a quojection Fréchet space and $\left\{S_{n}\right\}_{n=1}^{\infty} \subseteq$ $\mathscr{L}(X)$. Suppose that $X=\operatorname{proj}_{j}\left(X_{j}, Q_{j, j+1}\right)$, with $X_{j}$ a Banach space (having norm \|\|$_{j}$ ) and linking maps $Q_{j, j+1} \in$ $\mathscr{L}\left(X_{j+1}, X_{j}\right)$ which are surjective for all $j \in \mathbb{N}$, and suppose, for each $j, n \in \mathbb{N}$, that there exists $S_{n}^{(j)} \in \mathscr{L}\left(X_{j}\right)$ satisfying

$$
S_{n}^{(j)} Q_{j}=Q_{j} S_{n}
$$

where $Q_{j} \in \mathscr{L}\left(X, X_{j}\right), j \in \mathbb{N}$, denotes the canonical projection of $X$ onto $X_{j}$ (i.e., $Q_{j, j+1} \circ Q_{j+1}=Q_{j}$ ). Then the following statements are equivalent.

(i) The limit $\tau_{b}-\lim _{n \rightarrow \infty} S_{n}=: S$ exists in $\mathscr{L}_{b}(X)$.

(ii) For each $j \in \mathbb{N}$, the limit $\tau_{b}-\lim _{n \rightarrow \infty} S_{n}^{(j)}=: S^{(j)}$ exists in $\mathscr{L}_{b}\left(X_{j}\right)$.

In this case, the operators $S \in \mathscr{L}(X)$ and $S^{(j)} \in \mathscr{L}\left(X_{j}\right)$, for $j \in \mathbb{N}$, satisfy

$$
S x=\left(S^{(j)} x_{j}\right)_{j}, \quad x=\left(x_{j}\right)_{j} \in X .
$$

Moreover, (i) and (ii) remain equivalent if $\tau_{b}$ is replaced by $\tau_{s}$.

Given any lcHs $X$ and $T \in \mathscr{L}(X)$, let us introduce the notation:

$$
T_{[n]}:=\frac{1}{n} \sum_{m=1}^{n} T^{m}, \quad n \in \mathbb{N},
$$

for the Cesàro means of $T$. Then $T$ is called mean ergodic precisely when $\left\{T_{[n]}\right\}_{n=1}^{\infty}$ is a convergent sequence in $\mathscr{L}_{s}(X)$. If $\left\{T_{[n]}\right\}_{n=0}^{\infty}$ happens to be convergent in $\mathscr{L}_{b}(X)$, then $T$ will be called uniformly mean ergodic.

We always have the identities

$$
(I-T) T_{[n]}=T_{[n]}(I-T)=\frac{1}{n}\left(T-T^{n+1}\right), \quad n \in \mathbb{N},
$$


and also (setting $T_{[0]}:=I$ ) that

$$
\frac{1}{n} T^{n}=T_{[n]}-\frac{(n-1)}{n} T_{[n-1]}, \quad n \in \mathbb{N} .
$$

Some authors prefer to use $(1 / n) \sum_{m=0}^{n-1} T^{m}$ in place of $T_{[n]}$; since

$$
T_{[n]}=T\left(\frac{1}{n} \sum_{m=0}^{n-1} T^{m}\right)=\frac{1}{n}\left(T^{n}-I\right)+\frac{1}{n} \sum_{m=0}^{n-1} T^{m}, \quad n \in \mathbb{N},
$$

this leads to identical results.

Recall that $T \in \mathscr{L}(X)$ is called power bounded if $\left\{T^{n}\right\}_{n=1}^{\infty}$ is an equicontinuous subset of $\mathscr{L}(X)$.

The final result that we require (i.e., [18, Lemma 5.4]) is as follows.

Lemma 7. Let $X=\operatorname{proj}_{j}\left(X_{j}, Q_{j, j+1}\right)$ be a quojection Fréchet space and let operators $S \in \mathscr{L}(X)$ and $S_{j} \in \mathscr{L}\left(X_{j}\right)$, for $j \in \mathbb{N}$, be given which satisfy the assumptions of Lemma 5 (with $Q_{j} \in$ $\mathscr{L}\left(X, X_{j}\right), j \in \mathbb{N}$, denoting the canonical projection of $X$ onto $X_{j}$ and \|\|$_{j}$ being the norm in the Banach space $X_{j}$ ).

(i) $S \in \mathscr{L}(X)$ is power bounded if and only if each $S_{j} \in$ $\mathscr{L}\left(X_{j}\right), j \in \mathbb{N}$, is power bounded.

(ii) $S \in \mathscr{L}(X)$ is mean ergodic (resp., uniformly mean ergodic) if and only if each $S_{j} \in \mathscr{L}\left(X_{j}\right), j \in \mathbb{N}$, is mean ergodic (resp., uniformly mean ergodic).

\section{Spectrum, Uniform Convergence, and Mean Ergodicity}

A prequojection is a Fréchet space $X$ such that $X^{\prime \prime}$ is a quoection. Every quojection is a prequojection. A prequojection is called nontrivial if it is not itself a quojection. It is known that $X$ is a prequojection if and only if $X_{\beta}^{\prime}$ is a strict (LB) space. An alternative characterization is that $X$ is a prequojecton if and only if $X$ has no Köthe nuclear quotient which admits a continuous norm; see $[12,19-21]$. This implies that a quotient of a prequojection is again a prequojection. In particular, every complemented subspace of a prequojection is again a prequojection. The problem of the existence of nontrivial prequojections arose in a natural way in [12]; it has been solved, in the positive sense, in various papers $[19,22,23]$. All of these papers employ the same method, which consists in the construction of the dual of a prequojection, rather than the prequojection itself, which is often difficult to describe (see the survey paper [24] for further information). However, in [25] an alternative method for constructing prequojections is presented which has the advantage of being direct. For an example of a concrete space (i.e., a space of continuous functions on a suitable topological space), which is a nontrivial prequojection, see [26].

In this section we extend to prequojection Fréchet spaces some well-known results from the Banach setting which connect various conditions on the spectrum $\sigma(T)$, of a continuous linear operator $T$, to the operator norm convergence of certain sequences of operators generated by $T$. Such results have well-known consequences for the uniform mean ergodicity of $T$.
We begin with a construction for quojection Fréchet spaces which is needed in the sequel.

Let $X$ be a quojection Fréchet space and $\left\{q_{j}\right\}_{j=1}^{\infty}$ be any fundamental, increasing sequence of seminorms generating the lc-topology of $X$. For each $j \in \mathbb{N}$, set $X_{j}:=X / q_{j}^{-1}(\{0\})$ and endow $X_{j}$ with the quotient lc-topology. Denote by $Q_{j}: X \rightarrow$ $X_{j}$ the corresponding canonical (surjective) quotient map and define the quotient topology on $X_{j}$ via the increasing sequence of seminorms $\left\{\left(\widehat{q}_{j}\right)_{k}\right\}_{k=1}^{\infty}$ on $X_{j}$ by

$$
\begin{array}{r}
\left(\widehat{q}_{j}\right)_{k}\left(Q_{j} x\right):=\inf \left\{q_{k}(y): y \in X, Q_{j} y=Q_{j} x\right\}, \\
x \in X,
\end{array}
$$

for each $k \in \mathbb{N}$. Then

$$
\left(\widehat{q}_{j}\right)_{k}\left(Q_{j} x\right) \leq q_{k}(x), \quad x \in X, k, j \in \mathbb{N} .
$$

Moreover,

$$
\left(\widehat{q}_{j}\right)_{j}\left(Q_{j} x\right)=q_{j}(x), \quad x \in X, j \in \mathbb{N},
$$

which implies that $\left(\hat{q}_{j}\right)_{j}$ is a norm on $X_{j}$. As noted above, since $X$ is a quojection Fréchet space and every quotient space (of such a Fréchet space) with a continuous norm is necessarily Banach [12, Proposition 3], it follows that for each $j \in \mathbb{N}$ there exists $k(j) \geq j$ such that the norm $\left(\widehat{q}_{j}\right)_{k(j)}$ generates the lc-topology of $X_{j}$. Moreover, it is possible to choose $k(j+1) \geq$ $k(j)$ for all $j \in \mathbb{N}$. Thus, $X$ is isomorphic to the projective limit of the sequence $\left\{\left(X_{j},\left(\widehat{q}_{j}\right)_{k(j)}\right)\right\}_{j=1}^{\infty}$ of Banach spaces with respect to the continuous, surjective linking maps $Q_{j, j+1}$ : $X_{j+1} \rightarrow X_{j}$ defined by

$$
Q_{j, j+1} \circ Q_{j+1}=Q_{j}, \quad j \in \mathbb{N} .
$$

This particular construction will be used on various occasions in the sequel, where $\widehat{B}_{j}$ will always denote the closed unit ball of $X_{j}$, for $j \in \mathbb{N}$. The so-constructed Banach space norm $\left(\widehat{q}_{j}\right)_{k(j)}$ of $X_{j}$ will always be denoted by $\widetilde{q}_{j}$, for $j \in \mathbb{N}$.

The following result is classical in Banach spaces $[1$, p. 709 , Lemma 1].

Proposition 8. Let $X$ be a quojection Fréchet space and $T \in$ $\mathscr{L}(X)$ satisfy $\tau_{s}-\lim _{n \rightarrow \infty}\left(T^{n} / n\right)=0$. Then $\sigma(T) \subseteq \overline{\mathbb{D}}$.

In case $X$ is a prequojection Fréchet space and $\tau_{b}$ $\lim _{n \rightarrow \infty}\left(T^{n} / n\right)=0$, the inclusion $\sigma(T) \subseteq \overline{\mathbb{D}}$ is again valid.

Proof. We have the following two cases.

Case (I) ( $X$ is a quojection). Let $\left\{r_{j}\right\}_{j=1}^{\infty}$ be a fundamental, increasing sequence of seminorms generating the lc-topology of $X$. Since $T^{n} / n \rightarrow 0$ in $\mathscr{L}_{s}(X)$ as $n \rightarrow \infty$ and $X$ is a Fréchet space, the sequence $\left\{T^{n} / n\right\}_{n=1}^{\infty}$ is equicontinuous. So, for each $j \in \mathbb{N}$ there exists $c_{j}>0$ such that

$$
r_{j}\left(\frac{T^{n} x}{n}\right) \leq c_{j} r_{j+1}(x), \quad x \in X, n \in \mathbb{N}
$$

there is no loss in generality by assuming that $r_{j+1}$ can be chosen. 
Define $q_{j}$ on $X$ by $q_{j}(x):=\max \left\{r_{j}(x), \sup _{n \in \mathbb{N}} r_{j}\left(T^{n} x / n\right)\right\}$, for $x \in X$. Then (20) ensures that $\left\{q_{j}\right\}_{j=1}^{\infty}$ is also a fundamental, increasing sequence of seminorms generating the lctopology of $X$. Moreover,

$$
q_{j}(T x) \leq 2 q_{j}(x), \quad x \in X, j \in \mathbb{N} .
$$

We now apply the construction (16)-(19) to the sequence of seminorms $\left\{q_{j}\right\}_{j=1}^{\infty}$ to yield the corresponding sequence $\left\{\left(X_{j}, \widetilde{q}_{j}\right)\right\}_{j=1}^{\infty}$ of Banach spaces and the quotient maps $Q_{j} \epsilon$ $\mathscr{L}\left(X, X_{j}\right)$, for $j \in \mathbb{N}$; recall that $\widetilde{q}_{j}:=\left(\widehat{q}_{j}\right)_{k(j)}$, for $j \in \mathbb{N}$.

Fix $j \in \mathbb{N}$. Define the operator $T_{j}: X_{j} \rightarrow X_{j}$ via

$$
T_{j} Q_{j} x:=Q_{j} T x, \quad x \in X .
$$

Then $T_{j}$ is a well-defined, continuous linear operator from $X_{j}$ into $X_{j}$. Indeed, suppose $Q_{j} x=Q_{j} y$ for some $x, y \in X$; that is, $(x-y) \in \operatorname{Ker} Q_{j}$, so that $q_{j}(x-y)=0$. This, together with (21), yields $0 \leq q_{j}(T(x-y)) \leq 2 q_{j}(x-y)=0$. Since Ker $Q_{j}=$ $q_{j}^{-1}(\{0\})$, it follows that $Q_{j} T(x-y)=0$, and hence, by (22) that $T_{j} Q_{j}(x-y)=Q_{j} T(x-y)=0$. Therefore, $T_{j} Q_{j} x=T_{j} Q_{j} y$. This means that $T_{j}$ is well defined. Clearly, $T_{j}$ is also linear. Moreover, (17), (21), and (22) imply that

$$
\begin{aligned}
\tilde{q}_{j}\left(T_{j} \hat{x}\right) & =\tilde{q}_{j}\left(T_{j} Q_{j} x\right) \\
& =\tilde{q}_{j}\left(Q_{j} T x\right) \leq q_{k(j)}(T x) \leq 2 q_{k(j)}(x),
\end{aligned}
$$

for all $\widehat{x} \in X_{j}$ and $x \in X$ with $Q_{j} x=\widehat{x}$. Taking the infimum with respect to $x \in Q_{j}^{-1}(\{\widehat{x}\})$, it follows that

$$
\tilde{q}_{j}\left(T_{j} \hat{x}\right) \leq 2 \widetilde{q}_{j}(\widehat{x}), \quad \hat{x} \in X_{j} .
$$

Since $\tilde{q}_{j}$ generates the quotient topology of $X_{j}$, (24) ensures the continuity of $T_{j}$. Moreover, it follows from (22) that

$$
\left(T_{j}\right)^{n} Q_{j} x=Q_{j} T^{n} x, \quad x \in X, n \in \mathbb{N} .
$$

The surjectivity and the continuity of $Q_{j}$ together with (25) imply that $\tau_{s}-\lim _{n \rightarrow \infty}\left(\left(T_{j}\right)^{n} / n\right)=0$. Indeed, fix any $\hat{x} \in X_{j}$. By the surjectivity of $Q_{j}$ there exists $x \in X$ such that $Q_{j} x=\hat{x}$. By (25) it follows that $\left(T_{j}\right)^{n} \hat{x} / n=Q_{j}\left(T^{n} x / n\right)$, for $n \in \mathbb{N}$. Moreover, $T^{n} x / n \rightarrow 0$ as $n \rightarrow \infty$ by assumption. So, the continuity of $Q_{j}$ yields that $\lim _{n \rightarrow \infty}\left(\left(T_{j}\right)^{n} \hat{x} / n\right)=0$ in the Banach space $X_{j}$. We can then apply Lemma 1 in $[1, \mathrm{p} .709]$ to obtain that $\sigma\left(T_{j}\right) \subseteq \overline{\mathbb{D}}$.

We have just shown that $(\mathbb{C} \backslash \overline{\mathbb{D}}) \subseteq \cap_{j=1}^{\infty} \rho\left(T_{j}\right)$. Moreover, the operators $T$ and $T_{j}$ satisfy (22). So, we can apply Lemma 5 which yields $(\mathbb{C} \backslash \overline{\mathbb{D}}) \subseteq \rho(T)$; that is, $\sigma(T) \subseteq \overline{\mathbb{D}}$.

Case (II). ( $X$ is a prequojection and $\tau_{b}-\lim _{n \rightarrow \infty}\left(T^{n} / n\right)=$ $0)$. Observe that $X$ and $X_{\beta}^{\prime}$ are barrelled and, hence, quasibarrelled as $X$ is a Fréchet space and $X_{\beta}^{\prime}$ is the strong dual of a prequojection Fréchet space. Since $T^{\prime} \in \mathscr{L}\left(X_{\beta}^{\prime}\right)$ and $T^{\prime \prime} \in \mathscr{L}\left(X^{\prime \prime}\right)$, the condition $\tau_{b}-\lim _{n \rightarrow \infty}\left(T^{n} / n\right)=0$ implies that $\tau_{b}-\lim _{n \rightarrow \infty}\left(\left(T^{\prime \prime}\right)^{n} / n\right)=0$ (see [27, Lemma 2.6] or [28, Lemma 2.1]). On the other hand, $X^{\prime \prime}$ is a quojection Fréchet space. So, it follows from Case (I) that $\sigma\left(T^{\prime \prime}\right) \subseteq \overline{\mathbb{D}}$. Finally, Corollary 4 ensures that $\sigma(T)=\sigma\left(T^{\prime \prime}\right)$ and so $\sigma(T) \subseteq \overline{\mathbb{D}}$.
Remark 9. For a power-bounded operator $T \in \mathscr{L}(X)$ it is always the case that $\tau_{b}-\lim _{n \rightarrow \infty}\left(T^{n} / n\right)=0$ and so, whenever $X$ is a prequojection Fréchet space, it follows from Proposition 8 that $\sigma(T) \subseteq \overline{\mathbb{D}}$.

For operators in Banach spaces, the following result is due to Koliha [2].

Theorem 10. Let $X$ be a prequojection Fréchet space and $T \epsilon$ $\mathscr{L}(X)$. The following assertions are equivalent.

(i) $\tau_{b}-\lim _{n \rightarrow \infty} T^{n}=0$.

(ii) The series $\sum_{n=0}^{\infty} T^{n}$ converges in $\mathscr{L}_{b}(X)$.

(iii) $\tau_{b}-\lim _{n \rightarrow \infty}\left(T^{n} / n\right)=0$ and $\sigma(T) \subseteq \mathbb{D}$.

Moreover, if one (hence, all) of the above conditions holds, then $I-T$ is an isomorphism of $X$ onto $X$ with inverse $(I-T)^{-1}=$ $\sum_{n=0}^{\infty} T^{n}$ and the series converging in $\mathscr{L}_{b}(X)$.

Proof. We have the following two cases.

Case (I) ( $X$ is a quojection). (i) $\Rightarrow$ (ii). The assumption $\tau_{b^{-}}$ $\lim _{n \rightarrow \infty} T^{n}=0$ implies that $\tau_{b}-\lim _{n \rightarrow \infty}\left(T^{n} / n\right)=0$. So, we can proceed as in the proof of Proposition 8 to obtain that $X=\operatorname{proj}_{j}\left(X_{j}, Q_{j, j+1}\right)$ in such a way that, for every $j \in \mathbb{N}$, there exists $T_{j}$ in $\mathscr{L}\left(X_{j}\right)$ satisfying $T_{j} Q_{j}=Q_{j} T$. Then also $T_{j}^{n} Q_{j}=Q_{j} T^{n}$, for every $j, n \in \mathbb{N}$. So, Lemma 6 implies that $\tau_{b}-\lim _{n \rightarrow \infty} T_{j}^{n}=0$ for all $j \in \mathbb{N}$. Thus, by [2, Theorem 2.1] the series $\sum_{n=0}^{\infty} T_{j}^{n}$ converges in $\mathscr{L}_{b}\left(X_{j}\right)$, for each $j \in \mathbb{N}$. With $S_{n}:=\sum_{k=0}^{n} T^{k}$, for $n \in \mathbb{N}$, it follows again from Lemma 6 that the series $\sum_{n=0}^{\infty} T^{n}$ converges in $\mathscr{L}_{b}(X)$.

(ii) $\Rightarrow\left(\right.$ iii). The assumption clearly implies $\tau_{b^{-}}$ $\lim _{n \rightarrow \infty}\left(T^{n} / n\right)=0$. So, as in the proof of (i) $\Rightarrow$ (ii), we may assume that $X=\operatorname{proj}_{j}\left(X_{j}, Q_{j, j+1}\right)$ in such a way that, for every $j \in \mathbb{N}$, there exists $T_{j}$ in $\mathscr{L}\left(X_{j}\right)$ satisfying $T_{j} Q_{j}=Q_{j} T$. Then also $T_{j}^{n} Q_{j}=Q_{j} T^{n}$, for every $j, n \in \mathbb{N}$. Since $\sum_{n=0}^{\infty} T^{n}$ converges in $\mathscr{L}_{b}(X)$ and $X$ is a quojection, the series $\sum_{n=0}^{\infty} T_{j}^{n}$ also converges in $\mathscr{L}_{b}\left(X_{j}\right)$ for all $j \in \mathbb{N}$; see Lemma 6 . By [2, Theorem 2.1] we have that $\sigma\left(T_{j}\right) \subset \mathbb{D}$ and so $\Lambda:=$ $(\mathbb{C} \backslash \mathbb{D}) \subseteq \rho\left(T_{j}\right)$, for all $j \in \mathbb{N}$. Accordingly, since $T_{j} Q_{j}=Q_{j} T$ for all $j \in \mathbb{N}$, Lemma 5 yields $\Lambda \subseteq \cap_{j=1}^{\infty} \rho\left(T_{j}\right) \subseteq \rho(T)$; that is, $\sigma(T) \subset \mathbb{D}$.

(iii) $\Rightarrow$ (i). Since $\Lambda \subseteq \rho(T)$, for every $\lambda \in \Lambda$, the operator $I-\lambda^{-1} T=\lambda^{-1}(\lambda I-T) \in \mathscr{L}(X)$ is invertible, that is, bijective with $\left(I-\lambda^{-1} T\right)^{-1} \in \mathscr{L}(X)$. On the other hand, $\tau_{b}-\lim _{n \rightarrow \infty}\left(\left(\lambda^{-1} T\right)^{n} / n\right)=0$ for every $\lambda \in \Lambda$ as $\tau_{b^{-}}$ $\lim _{n \rightarrow \infty}\left(T^{n} / n\right)=0$ and $\left|\lambda^{-1}\right| \leq 1$. So, by Theorem 4.1 in [29] (see also Theorem 3.5 of [6]) we can conclude that

$$
\tau_{b}-\lim _{n \rightarrow \infty}\left(\lambda^{-1} T\right)_{[n]}=0, \quad \lambda \in \Lambda .
$$

Let $\left\{r_{j}\right\}_{j=1}^{\infty}$ be a fundamental, increasing sequence of seminorms generating the lc-topology of $X$. Arguing as in the proof of Proposition 8 (and adopting the notation from there) we conclude that (20) is satisfied. Define $q_{j}$ on $X$ by $q_{j}(x):=$ $\max \left\{r_{j}(x), \sup _{n \in \mathbb{N}} r_{j}\left(T^{n} x / n\right)\right\}$, for $x \in X$. Then again (21) is satisfied and, for each $j \in \mathbb{N}$, there exists a continuous 
linear operator $T_{j}: X_{j} \rightarrow X_{j}$ satisfying both (22) and (24). Moreover, it follows from (22) that

$$
\left(\lambda^{-1} T_{j}\right)^{n} Q_{j} x:=Q_{j}\left(\lambda^{-1} T\right)^{n} x, \quad x \in X, n \in \mathbb{N}, \lambda \in \Lambda .
$$

Fix $\lambda \in \Lambda$ and consider the sequences $\left\{R_{n}\right\}_{n=1}^{\infty}$ and $\left\{H_{n}\right\}_{n=1}^{\infty}$ in $\mathscr{L}(X)$ given by $R_{n}:=(1 / n) \sum_{m=0}^{n-1} \sum_{h=0}^{m}\left(\lambda^{-1} T\right)^{h}$ and $H_{n}:=$ $I-\left(\lambda^{-1} T\right)_{[n]}$, for $n \in \mathbb{N}$. Then the operator $A:=I-\lambda^{-1} T$ satisfies $H_{n}=A R_{n}=R_{n} A$ for all $n \in \mathbb{N}$. Moreover, (26) implies that $H_{n} \rightarrow I$ in $\mathscr{L}_{b}(X)$. Since all the assumptions of Lemma 3.4 in [6] are satisfied with $F=E=X, R=I \in \mathscr{L}(X$, $X)$, and $A=I-\lambda^{-1} T$, we can proceed as in the proof of that result to conclude, for every $j \in \mathbb{N}$, that the operator $I-\lambda^{-1} T_{j}$ is invertible in $\mathscr{L}\left(X_{j}\right)$ (hence, also $\lambda I-T_{j}$ is invertible); that is, $\lambda \in \rho\left(T_{j}\right)$.

By the arbitrariness of $\lambda \in \Lambda$, we have that $\Lambda \subseteq \rho\left(T_{j}\right)$, for all $j \in \mathbb{N}$. So, there exists $\delta_{j} \in(0,1)$ such that $\rho\left(T_{j}\right) \supset\{\lambda \in$ $\left.\mathbb{C}:|\lambda| \geq 1-\delta_{j}\right\}$. It follows that

$$
\begin{aligned}
r\left(T_{j}\right) & :=\max \left\{|\lambda|: \lambda \in \sigma\left(T_{j}\right)\right\} \\
& =\lim _{n \rightarrow \infty} \sqrt[n]{\left\|T_{j}^{n}\right\|_{\text {op }}} \leq\left(1-\delta_{j}\right)<1, \quad j \in \mathbb{N},
\end{aligned}
$$

and, hence, that $\lim _{n \rightarrow \infty}\left\|T_{j}^{n}\right\|_{\text {op }}=0$. Because of (27), with $\lambda=1 \in \Lambda$, it follows from Lemma 6 (with $S_{n}:=T^{n}$ ) that $\tau_{b^{-}}$ $\lim _{n \rightarrow \infty} T^{n}=0$.

Case (II) ( $X$ is a prequojection). As noted before $X$ and $X_{\beta}^{\prime}$ are barrelled with $T^{\prime} \in \mathscr{L}\left(X_{\beta}^{\prime}\right)$ and $T^{\prime \prime} \in \mathscr{L}\left(X^{\prime \prime}\right)$.

(i) $\Rightarrow$ (ii). If $T^{n} \rightarrow 0$ in $\mathscr{L}_{b}(X)$ for $n \rightarrow \infty$, then an argument as for Case (II) in the proof of Proposition 8 shows that $\left(T^{\prime \prime}\right)^{n}=\left(T^{n}\right)^{\prime \prime} \rightarrow 0$ in $\mathscr{L}_{b}\left(X^{\prime \prime}\right)$ for $n \rightarrow \infty$. Since $X^{\prime \prime}$ is a quojection Fréchet space, we can apply (i) $\Rightarrow$ (ii) of Case (I) above to conclude that the series $\sum_{n=0}^{\infty}\left(T^{\prime \prime}\right)^{n}$ converges in $\mathscr{L}_{b}\left(X^{\prime \prime}\right)$. Then also $\sum_{n=0}^{\infty} T^{n}$ converges in $\mathscr{L}_{b}(X)$ as $\left.T^{\prime \prime}\right|_{X}=T$ and $X$ is a closed subspace of $X^{\prime \prime}$.

(ii) $\Rightarrow$ (iii). If $\sum_{n=0}^{\infty} T^{n}$ converges in $\mathscr{L}_{b}(X)$, then $\sum_{n=0}^{\infty}\left(T^{\prime \prime}\right)^{n}$ converges in $\mathscr{L}_{b}\left(X^{\prime \prime}\right)$; see [27, Lemma 2.6] or [28, Lemma 2.1]. Since $X^{\prime \prime}$ is a quojection Fréchet space, we can apply (ii) $\Rightarrow$ (iii) of Case (I) above to conclude that $\sigma\left(T^{\prime \prime}\right) \subset \mathbb{D}$ (the condition $\tau_{b}-\lim _{n \rightarrow \infty}\left(T^{n} / n\right)=0$ clearly follows from the assumption). So, $\sigma(T) \subseteq \mathbb{D}$ by Corollary 4 .

(iii) $\Rightarrow$ (i). As already noted (cf. proof of Case (II) in Proposition 8) $X$ and $X_{\beta}^{\prime}$ are barrelled (hence, quasibarrelled) and $\tau_{b}-\lim _{n \rightarrow \infty}\left(\left(T^{\prime \prime}\right)^{n} / n\right)=0$. By Corollary 4 , $\rho\left(T^{\prime \prime}\right)=\rho(T)$ and so $\Lambda \subseteq \rho\left(T^{\prime \prime}\right)$ by assumption. Since $X^{\prime \prime}$ is a quojection Fréchet space, we can apply Case (I) to conclude that $\tau_{b}-\lim _{n \rightarrow \infty}\left(T^{\prime \prime}\right)^{n}=0$. So, also $\tau_{b}-\lim _{n \rightarrow \infty} T^{n}=0$ as $\left.T^{\prime \prime}\right|_{X}=T$ and $X$ is a closed subspace of $X^{\prime \prime}$.

Finally, suppose that one (hence, all) of the above conditions holds. Then the series $\sum_{n=0}^{\infty} T^{n}$ converges in $\mathscr{L}_{b}(X)$ and so $T^{n} \rightarrow 0$ in $\mathscr{L}_{b}(X)$ for $n \rightarrow \infty$. But, for every $n \in \mathbb{N}$, we have

$$
(I-T) \sum_{m=0}^{n} T^{m}=\sum_{m=0}^{n}\left(T^{m}-T^{m+1}\right)=\left(I-T^{n+1}\right)
$$

and so, for $n \rightarrow \infty$, we can conclude that $(I-T) \sum_{n=0}^{\infty} T^{n}=$ $I$ with convergence of the series in $\mathscr{L}_{b}(X)$. In a similar way one shows that $\left(\sum_{n=0}^{\infty} T^{n}\right)(I-T)=I$, with the series again converging in $\mathscr{L}_{b}(X)$.

Remark 11. In the proof of (iii) $\Rightarrow$ (i) in Case (I) above, if $\inf _{j \in \mathbb{N}} \delta_{j}=: \delta>0$, then it follows that $\rho(T) \supset\{\lambda \in \mathbb{C}:|\lambda| \geq$ $(1-\delta)\}$. But, this is not the case in general as the following example shows.

Let $X$ be a Banach space and let $\left\{\lambda_{n}\right\}_{n=1}^{\infty} \in(0,1)$ be an increasing sequence with $\sup _{n \in \mathbb{N}} \lambda_{n}=1$. Consider the quojection Fréchet space $X^{\mathbb{N}}$ (endowed with the product topology) and the operator $T$ on $X^{\mathbb{N}}$ defined by $T\left(x_{n}\right)_{n}:=\left(\lambda_{n} x_{n}\right)_{n}$, for $\left(x_{n}\right)_{n} \in X^{\mathbb{N}}$. It is easy to show that $T \in \mathscr{L}(X)$ and that $T$ is even power bounded. Moreover, $\Lambda \subseteq \rho(T)$. Indeed, for a fixed $\lambda \in \Lambda$, if $x \in \operatorname{Ker}(\lambda I-T)$, then $\lambda x-T x=0$; that is, $(\lambda-$ $\left.\lambda_{n}\right) x_{n}=0$ for all $n \in \mathbb{N}$. Since $\lambda \notin\left\{\lambda_{n}\right\}_{n=1}^{\infty}$, it follows that $x_{n}=0$ for all $n \in \mathbb{N}$ and so $x=0$. On the other hand, if $y \in X^{\mathbb{N}}$, then $x:=\left(y_{n} /\left(\lambda-\lambda_{n}\right)\right)_{n}$ belongs to $X^{\mathbb{N}}$ and $T x=y$. Hence, $\lambda I-T$ is bijective and so $\lambda \in \rho(T)$. Moreover, fix any $x \in X \backslash\{0\}$ and set $e_{n}:=\left(\delta_{n m} x\right)_{m}$ for every $n \in \mathbb{N}$. Then $T e_{n}=$ $\lambda_{n} e_{n}$ for every $n \in \mathbb{N}$. Thus, each $\lambda_{n}$ is an eigenvalue of $T$.

Now, suppose that $\rho(T) \supset\{\lambda \in \mathbb{C}:|\lambda| \geq 1-\delta\}$ for some $\delta \in(0,1)$. Then $B(1, \delta / 2):=\{\mu \in \mathbb{C}:|\mu-1|<\delta / 2\} \subset \rho(T)$. But $\lambda_{n} \rightarrow 1$ for $n \rightarrow \infty$, and hence, there is $n_{0} \in \mathbb{N}$ such that $\lambda_{n_{0}} \in B(1, \delta / 2) \subset \rho(T)$. This contradiction as $\lambda_{n_{0}}$ is an eigenvalue for $T$.

If $T$ is uniformly mean ergodic, then (14) implies that $\tau_{b}$ $\lim _{n \rightarrow \infty}\left(T^{n} / n\right)=0$. With an extra condition the converse is also valid.

Corollary 12. Let $X$ be a prequojection Fréchet space and $T \in \mathscr{L}(X)$. If $\tau_{b}-\lim _{n \rightarrow \infty}\left(T^{n} / n\right)=0$ and $1 \in \rho(T)$, then $T$ is uniformly mean ergodic.

Proof. Since $1 \in \rho(T)$, the operator $I-T$ is bijective and so the space $(I-T)(X)=X$ is closed in $X$. By [6, Theorem 3.5], $T$ is uniformly mean ergodic. In particular, as $\operatorname{Ker}(I-T)=\{0\}$, we have that $T_{[n]} \rightarrow 0$ in $\mathscr{L}_{b}(X)$ for $n \rightarrow \infty$.

Remark 13. Let $X$ be a prequojection Fréchet space and let $T \in \mathscr{L}(X)$ satisfy $\tau_{b}-\lim _{n \rightarrow \infty}\left(T^{n} / n\right)=0$. If $1 \in \rho(T)$, then the proof of Corollary 12 shows that $T$ is uniformly mean ergodic with $\tau_{b}-\lim _{n \rightarrow \infty} T_{[n]}=0$. On the other hand, if $\sigma(T) \subseteq \mathbb{D}$ (a stronger condition than $1 \in \rho(T))$, then Theorem 10 implies that $\tau_{b}-\lim _{n \rightarrow \infty} T^{n}=0$ and hence again $\tau_{b}-\lim _{n \rightarrow \infty} T_{[n]}=0$ follows [30, Remark 3.1]. However, the stronger conclusion that $\tau_{b}-\lim _{n \rightarrow \infty} T^{n}=0$ does not follow from Corollary 12 in general. Indeed, let $X \neq\{0\}$ be any Banach space (even finite dimensional). Then every power of $T:=$ iI belongs to the set $\{-I, I,-i I, i I\}$ and so $T$ is power bounded. This implies that $\tau_{b}-\lim _{n \rightarrow \infty}\left(T^{n} / n\right)=0$. Since $\sigma(T)=\{i\}$, surely $1 \in \rho(T)$ and so, by Corollary 12 , it follows that $\tau_{b^{-}}$ $\lim _{n \rightarrow \infty} T_{[n]}=0$. However, for every $n \in \mathbb{N}$ we have $\left\|T^{n}\right\|_{\text {op }}=$ 1 and so $\left\{\left\|T^{n}\right\|_{\text {op }}\right\}_{n=1}^{\infty}$ does not converge to zero. This does not contradict Theorem 10 as $\sigma(T)$ is not included in $\mathbb{D}$.

Remark 14. Let $X$ be a prequojection Fréchet space and $T \epsilon$ $\mathscr{L}(X)$. We observe the following. 
(i) Proposition 8 and (14) yield that if $T$ is uniformly mean ergodic, then $\tau_{b}-\lim _{n \rightarrow \infty}\left(T^{n} / n\right)=0$ and $\sigma(T) \subseteq \overline{\mathbb{D}}$.

(ii) Suppose that $\tau_{b}-\lim _{n \rightarrow \infty}\left(T^{n} / n\right)=0$. If $\sigma(T) \subseteq$ $\mathbb{D}$, then $T$ is uniformly mean ergodic and $\tau_{b}$ $\lim _{n \rightarrow \infty} T_{[n]}=0$ (cf. Remark 13).

For Banach spaces the next result is due to Mbekhta and Zemànek [3]. Recall that $\Gamma(T):=\sigma(T) \cap \mathbb{T}$.

Theorem 15. Let $X$ be a prequojection Fréchet space and $T \in$ $\mathscr{L}(X)$. The following statements are equivalent.

(i) $\left\{T^{n}\right\}_{n=1}^{\infty}$ is convergent in $\mathscr{L}_{b}(X)$.

(ii) $\tau_{b}-\lim _{n \rightarrow \infty}\left(T^{n} / n\right)=0$, the linear space $(I-T)^{m}(X)$ is closed in $X$ for some $m \in \mathbb{N}$ and $\Gamma(T) \subseteq\{1\}$.

(iii) $\tau_{b}-\lim _{n \rightarrow \infty}\left(T^{n}-T^{n+1}\right)=0$ and $(I-T)^{m}(X)$ is closed for some $m \in \mathbb{N}$.

Proof. (i) $\Rightarrow$ (ii). If $\left\{T^{n}\right\}_{n=1}^{\infty}$ converges in $\mathscr{L}_{b}(X)$ to $P$, say, then $T$ is uniformly mean ergodic with ergodic projection equal to $P$ [30, Remark 3.1]. Moreover, as $\left\{T^{n}\right\}_{n=1}^{\infty}$ is necessarily equicontinuous, it follows that $\tau_{b}-\lim _{n \rightarrow \infty}\left(T^{n} / n\right)=0$. Hence, by Theorem 3.5 and Remark 3.6 of [6] the space $(I-T)^{m}(X)$ is closed for every $m \in \mathbb{N}$. Moreover, by Proposition 8 we have $\sigma(T) \subseteq \overline{\mathbb{D}}$. To establish the remaining condition $\Gamma(T) \subseteq\{1\}$ we distinguish two cases.

(a) $X$ is a quojection. Let $\left\{r_{j}\right\}_{j=1}^{\infty}$ be any fundamental, increasing sequence of seminorms generating the lc-topology of $X$. By equicontinuity of $\left\{T^{n}\right\}_{n=1}^{\infty}$, for each $j \in \mathbb{N}$, there exists $c_{j}>0$ such that

$$
r_{j}\left(T^{n} x\right) \leq c_{j} r_{j+1}(x), \quad x \in X, n \in \mathbb{N} .
$$

Define $q_{j}$, for each $j \in \mathbb{N}$, by $q_{j}(x):=\sup _{n \geq 0} r_{j}\left(T^{n} x\right)$, for $x \in X$. Then (30) ensures that $\left\{q_{j}\right\}_{j=1}^{\infty}$ is also a fundamental, increasing sequence of seminorms generating the lc-topology of $X$. Moreover, it is routine to check (using also that $T^{n} x \rightarrow$ $P x$ for each $x \in X)$ that

$$
q_{j}(T x) \leq q_{j}(x), \quad q_{j}(P x) \leq q_{j}(x), \quad x \in X, j \in \mathbb{N} .
$$

With (31) in place of (21), we can argue as in the proof of Proposition 8 to deduce that $X=\operatorname{proj}_{j}\left(X_{j}, Q_{j, j+1}\right)$ and that, for every $j \in \mathbb{N}$, there exist operators $T_{j}$ and $P_{j}$ in $\mathscr{L}\left(X_{j}\right)$ satisfying $T_{j} Q_{j}=Q_{j} T$ and $P_{j} Q_{j}=Q_{j} P$. Hence, $T_{j}^{n} Q_{j}=Q_{j} T^{n}$ for every $j, n \in \mathbb{N}$. Since also $\tau_{b}-\lim _{n \rightarrow \infty} T^{n}=P$, it follows from Lemma 6 (with $S_{n}:=T^{n}$ and $S:=P$ ) that $\tau_{b}-\lim _{n \rightarrow \infty} T_{j}^{n}=P_{j}$, for each $j \in \mathbb{N}$. By [3, Corollaire 3] we have that $\Gamma\left(T_{j}\right) \subseteq\{1\}$ for every $j \in \mathbb{N}$. This implies that $\Gamma(T) \subseteq\{1\}$. Indeed, if $\lambda \in \mathbb{T} \backslash\{1\}$, then for every $j \in \mathbb{N}$ we have $\lambda \notin \Gamma\left(T_{j}\right)$ and so $\lambda \in \rho\left(T_{j}\right)$; that is, $\lambda \in \cap_{j=1}^{\infty} \rho\left(T_{j}\right)$. As $T_{j} Q_{j}=Q_{j} T$ for every $j \in \mathbb{N}$, an appeal to Lemma 5 yields that $\lambda \in \rho(T)$.

(b) $X$ is a prequojection. As noted before, $X$ and $X_{\beta}^{\prime}$ are barrelled (hence, quasi-barrelled) with $T^{\prime}, P^{\prime} \in \mathscr{L}\left(X_{\beta}^{\prime}\right)$ and $T^{\prime \prime}, P^{\prime \prime} \in \mathscr{L}\left(X^{\prime \prime}\right)$. Hence, $\tau_{b}-\lim _{n \rightarrow \infty} T^{n}=P$ implies that $\tau_{b^{-}}$ $\lim _{n \rightarrow \infty}\left(T^{\prime \prime}\right)^{n}=P^{\prime \prime}$; see [27, Lemma 2.6] or [28, Lemma 2.1].
Since $X^{\prime \prime}$ is a quojection Fréchet space, we can apply the result from case (a) to conclude that $\Gamma\left(T^{\prime \prime}\right) \subseteq\{1\}$ and so $\Gamma(T) \subseteq\{1\}$; see Corollary 4.

(ii) $\Rightarrow$ (i). The assumptions $\tau_{b}-\lim _{n \rightarrow \infty}\left(T^{n} / n\right)=0$ and the space $(I-T)^{m}(X)$ being closed for some $m \in \mathbb{N}$ imply that $T$ is uniformly mean ergodic [6, Theorem 3.4 and Remark 3.6]. In particular, $(I-T)(X)$ is closed and

$$
X=\operatorname{Ker}(I-T) \oplus(I-T)(X)
$$

[6, Theorem 3.4]. Moreover, Proposition 8 implies that $\sigma(T) \subseteq \overline{\mathbb{D}}$. It then follows from the assumption $\Gamma(T) \subseteq\{1\}$ that either $\Gamma(T)=\emptyset$ or $\Gamma(T)=\{1\}$.

If $\Gamma(T)=\emptyset$, then necessarily $\sigma(T) \subseteq \mathbb{D}$ and so, by (iii) $\Rightarrow$ (i) of Theorem 10, we have $\tau_{b}-\lim _{n \rightarrow \infty} T^{n}=0$.

In the event that $\Gamma(T)=\{1\}$ we have that $1 \in \sigma(T)$ and so $\operatorname{Ker}(I-T) \neq\{0\}$ (otherwise, $(I-T)$ is injective and from $X=\operatorname{Ker}(I-T) \oplus(I-T)(X)=(I-T)(X)$ also surjective; that is, $1 \in \rho(T))$. Define $Y:=(I-T)(X)$ and $T_{1}:=\left.T\right|_{Y}$. Then $Y$ is a prequojection Fréchet space (being a quotient space of the prequojection $X$ ) which is $T$-invariant and so $T_{1} \in \mathscr{L}(Y)$. The claim is that

$$
\rho\left(T_{1}\right)=\rho(T) \cup\{1\} .
$$

It follows from (32) that $1 \in \rho\left(T_{1}\right)$. Fix $\lambda \in \rho(T)$ (so that $\lambda \neq 1)$. If $\left(\lambda I-T_{1}\right) x=0$ for some $x \in Y$ (i.e., $\left.(\lambda I-T) x=0\right)$, then $x=0$ as $\lambda \in \rho(T)$. Hence, $\left(\lambda I-T_{1}\right)$ is injective. Next, let $y \in Y$. Then there exists $x \in X$ such that $(\lambda I-T) x=y$. Since $x=x_{1}+x_{2}$ with $x_{1} \in \operatorname{Ker}(I-T)$ and $x_{2} \in Y$ (cf. (32)), it follows that $(\lambda-1) x_{1}+\left(\lambda I-T_{1}\right) x_{2}=y$; that is, $(\lambda-1) x_{1}=y-\left(\lambda I-T_{1}\right) x_{2}$, with $(\lambda-1) x_{1} \in \operatorname{Ker}(I-T)$ and $\left(y-\left(\lambda I-T_{1}\right) x_{2}\right) \in Y$. As $\operatorname{Ker}(I-T) \cap Y=\{0\}$ and $\lambda \neq 1$, this implies that $x_{1}=0$ and so $\left(\lambda I-T_{1}\right) x_{2}=y$ with $x_{2} \in Y$; that is, $\left(\lambda I-T_{1}\right)$ is surjective. These facts show that $\lambda \in \rho\left(T_{1}\right)$. This establishes $\rho(T) \cup\{1\} \subseteq \rho\left(T_{1}\right)$.

Fix $\lambda \in \rho\left(T_{1}\right) \backslash\{1\}$. Suppose that $(\lambda I-T) x=0$ for some $x \in X$. Then $x=x_{1}+x_{2}$ with $x_{1} \in \operatorname{Ker}(I-T)$ and $x_{2} \in Y$ (cf. (32)). It follows that $(\lambda-1) x_{1}+\left(\lambda I-T_{1}\right) x_{2}=0$ with $(\lambda-1) x_{1} \in$ $\operatorname{Ker}(I-T)$ and $\left(\lambda I-T_{1}\right) x_{2} \in Y$. Arguing as in the previous paragraph, this implies that $x_{1}=0$ and $\left(\lambda I-T_{1}\right) x_{2}=0$. Since $x_{2} \in Y$ and $\lambda \in \rho\left(T_{1}\right)$, we can conclude that $x=0$; that is, $(\lambda I-T)$ is injective. Next, let $y \in X$. Then $y=y_{1}+y_{2}$ with $y_{1} \in \operatorname{Ker}(I-T)$ and $y_{2} \in Y$ (cf. (32)). Since $\lambda \neq 1$, the element $x_{1}:=y_{1} /(\lambda-1) \in \operatorname{Ker}(I-T)$ exists. Moreover, $\lambda \in \rho\left(T_{1}\right)$ with $y_{2} \in Y$ implies the existence of $x_{2} \in Y$ such that $y_{2}=(\lambda I-$ $\left.T_{1}\right) x_{2}=(\lambda I-T) x_{2}$. It follows that $x:=\left(x_{1}+x_{2}\right) \in X$ satisfies $(\lambda I-T) x=y$. Hence, $(\lambda I-T)$ is also surjective and so $\lambda \epsilon$ $\rho(T)$. Accordingly, $\rho\left(T_{1}\right) \subseteq \rho(T) \cup\{1\}$ is proved. This establishes (33).

Since $\sigma(T) \subseteq \mathbb{D} \cup\{1\}$ and (33) is equivalent to $\sigma\left(T_{1}\right)=$ $\sigma(T) \backslash\{1\}$, it follows that $\sigma\left(T_{1}\right) \subseteq \mathbb{D}$. Moreover, $Y$ is a prequojection Fréchet space and $\left(T_{1}\right)^{n} / n \rightarrow 0$ in $\mathscr{L}_{b}(Y)$ as $n \rightarrow \infty$ (because $\tau_{b}-\lim _{n \rightarrow \infty}\left(T^{n} / n\right)=0$ and $T_{1}=T$ on $Y$ ). So, we can apply Theorem 10 to conclude that $T_{1}^{n} \rightarrow 0$ in $\mathscr{L}_{b}(Y)$ as $n \rightarrow \infty$. On the other hand, $T=I$ on $\operatorname{Ker}(I-T)$. These facts ensure that $T^{n}=I \oplus\left(T_{1}\right)^{n} \rightarrow I \oplus 0$ in $\mathscr{L}_{b}(X)$ because $X=$ $\operatorname{Ker}(I-T) \oplus Y$ and $T_{1}=T$ on $Y$.

(i) $\Rightarrow$ (iii). If $\left\{T^{n}\right\}_{n=1}^{\infty}$ converges to some $P$ in $\mathscr{L}_{b}(X)$, then $T$ is uniformly mean ergodic with ergodic projection equal to $P$ 
[30, Remark 3.1]. Hence, by [6, Theorem 3.5 and Remark 3.6] the space $(I-T)^{m}(X)$ is closed for every $m \in \mathbb{N}$. Moreover, $\left(T^{n}-T^{n+1}\right) \rightarrow P-P=0$ in $\mathscr{L}_{b}(X)$ as $n \rightarrow \infty$.

(iii) $\Rightarrow$ (i). We first observe that

$$
\frac{1}{n} \sum_{m=1}^{n}\left(T^{m}-T^{m+1}\right)=\frac{1}{n}\left(T-T^{n+1}\right), \quad n \in \mathbb{N} .
$$

This identity (together with the fact that $\tau_{b}-\lim _{n \rightarrow \infty}\left(T^{n}-\right.$ $\left.T^{n+1}\right)=0$ implies for the averages that $\tau_{b}$ - $\lim _{n \rightarrow \infty}(1 / n)$ $\sum_{m=1}^{n}\left(T^{m}-T^{m+1}\right)=0\left[30\right.$, Remark 3.1]) yields $\tau_{b}-\lim _{n \rightarrow \infty}(1 /$ $n)\left(T-T^{n+1}\right)=0$. But, $\tau_{b}-\lim _{n \rightarrow \infty}(T / n)=0$ and so we can conclude that $\tau_{b}-\lim _{n \rightarrow \infty}\left(T^{n} / n\right)=0$. As also $(I-T)^{m}(X)$ is closed for some $m \in \mathbb{N}$, we can apply [6, Theorem 3.4 and Remark 3.6] to conclude that $T$ is uniformly mean ergodic and, in particular, that $(32)$ is valid with $(I-T)(X)$ being closed. We claim that this fact, together with the assumption that $\tau_{b}-\lim _{n \rightarrow \infty}\left(T^{n}-T^{n+1}\right)=0$, implies that $\left\{T^{n}\right\}_{n=1}^{\infty}$ converges in $\mathscr{L}_{b}(X)$. To see this, note that $T=I$ on $\operatorname{Ker}(I-T)$ and so $T^{n}=I \rightarrow I$ in $\mathscr{L}_{b}(\operatorname{Ker}(I-T))$ as $n \rightarrow \infty$. On the other hand, the surjective operator $(I-T): X \rightarrow(I-T)(X)$ lifts bounded sets via [10, Lemma 26.13] because $X$ and $\operatorname{Ker}(I-T)$, both being prequojections, are quasinormable Fréchet spaces [24, Proposition 2.1], [21]; that is, for every $C \in \mathscr{B}((I-T)(X))$ there exists $B \in \mathscr{B}(X)$ such that $C \subseteq(I-T)(B)$. So, for fixed $C \in \mathscr{B}((I-T)(X))$ (with corresponding set $B \in \mathscr{B}(X))$ and $p \in \Gamma_{X}$ (every $q \in \Gamma_{(I-T) X}$ is the restriction of some $p \in \Gamma_{X}$ ), we have

$$
\begin{aligned}
\sup _{y \in C} p\left(T^{n} y\right) & \leq \sup _{x \in B} p\left(T^{n}(I-T) x\right) \\
& =\sup _{x \in B} p\left(\left(T^{n}-T^{n+1}\right) x\right), \quad n \in \mathbb{N},
\end{aligned}
$$

where $\sup _{x \in B} p\left(\left(T^{n}-T^{n+1}\right) x\right) \rightarrow 0$ as $n \rightarrow \infty$ by assumption. Set $T_{1}:=\left.T\right|_{(I-T)(X)}$. The arbitrariness of $C$ and $p$ shows that $\left(T_{1}\right)^{n} \rightarrow 0$ in $\mathscr{L}_{b}((I-T)(X))$ (after observing that $(I-$ $T)(X)$ is $T$-invariant and so $\left.T_{1}=\left.T\right|_{(I-T)(X)} \in \mathscr{L}((I-T)(X))\right)$. These facts ensure that $T^{n}=I \oplus\left(T_{1}\right)^{n} \rightarrow I \oplus 0$ in $\mathscr{L}_{b}(X)$ as $X=\operatorname{Ker}(I-T) \oplus Y$.

Remark 16. In assertion (ii) of Theorem 15 the condition that " $(I-T)^{m}(X)$ is closed in $X$ for some $m \in \mathbb{N}$ " can be replaced with the condition that " $T$ is uniformly mean ergodic"; see [6, Theorem 3.5 and Remark 3.6].

Theorems 10 and 15 do not necessarily hold for operators acting in general Fréchet spaces.

Proposition 17. Let $p \in[1, \infty)$ or $p=0$ and let $A$ be $a$ Köthe matrix on $\mathbb{N}$ such that $\lambda_{p}(A)$ is a Montel space with $\lambda_{p}(A) \neq \mathbb{C}^{\mathbb{N}}$. Then there exists an operator $T \in \mathscr{L}\left(\lambda_{p}(A)\right)$ such that $T^{n} \rightarrow 0$ in $\mathscr{L}_{b}\left(\lambda_{p}(A)\right)$ as $n \rightarrow \infty$ and $\Gamma(T)=\{1\}$ but $(I-T)^{m}\left(\lambda_{p}(A)\right)$ is not closed for every $m \in \mathbb{N}$.

Proof. By the proof of Proposition 3.1 in [6] there exists $d:=$ $\left(d_{i}\right)_{i} \in \mathbb{R}^{\mathbb{N}}$ with $0<d_{i}<1$ for all $i \in \mathbb{N}$ such that the diagonal operator $T: \lambda_{p}(A) \rightarrow \lambda_{p}(A)$ given by $T\left(\left(x_{i}\right)_{i}\right):=\left(d_{i} x_{i}\right)_{i}$, for $x=\left(x_{i}\right)_{i} \in \lambda_{p}(A)$, is power bounded, uniformly mean ergodic and $(I-T)\left(\lambda_{p}(A)\right)$ is dense but, not closed in $\lambda_{p}(A)$. So, for every $m \in \mathbb{N}$, also $(I-T)^{m}\left(\lambda_{p}(A)\right)$ is dense but not closed in $\lambda_{p}(A)$. To see this, note that the arguments in the proof of $[6$, Remark 3.6, $(5) \Rightarrow(4)$ ] are valid for any operator $T$ satisfying $\tau_{s}-\lim _{n \rightarrow \infty}\left(T^{n} / n\right)=0$ and acting in any Fréchet space. So, in the case that $(I-T)^{m}\left(\lambda_{p}(A)\right)$ was closed for some $m \in$ $\mathbb{N}$, we could apply $[6$, Remark $3.6,(5) \Rightarrow(4)]$ to conclude that $(I-T)\left(\lambda_{p}(A)\right)$ is also closed; a contradiction. So $1 \in \Gamma(T)$.

We claim that $T^{n} \rightarrow 0$ in $\mathscr{L}_{b}\left(\lambda_{p}(A)\right)$ as $n \rightarrow \infty$. Indeed, since $\left\{T^{n}\right\}_{n=1}^{\infty}$ is equicontinuous and convergence of a sequence in $\mathscr{L}_{b}\left(\lambda_{p}(A)\right)$ is equivalent to its convergence in $\mathscr{L}_{s}\left(\lambda_{p}(A)\right)$ (as $\lambda_{p}(A)$ is Montel), it suffices to show that $\lim _{n \rightarrow \infty} T^{n} e_{j}=0$ in $\lambda_{p}(A)$ for each $j \in \mathbb{N}$, where $e_{j}:=\left(\delta_{i j}\right)_{i} \in$ $\lambda_{p}(A)$. But, this is immediate because $T^{n} e_{j}=d_{j}^{n} e_{j}$, for all $j, n \in \mathbb{N}$.

It remains to show that $\Gamma(T) \subseteq\{1\}$. Set $D:=\overline{\left\{d_{i}: i \in \mathbb{N}\right\}}$. Then $D \subseteq[0,1]$. Let $\lambda \in \mathbb{T} \backslash\{1\}$. Then $\inf _{i \in \mathbb{N}}\left|\lambda-d_{i}\right|=$ : $\delta>0$. It is routine to check that, for a fixed $y \in \lambda_{p}(A)$, the element $x:=\left(\left(1 /\left(\lambda-d_{i}\right)\right) y_{i}\right)_{i}$ belongs to $\lambda_{p}(A)$ and satisfies $(\lambda I-T) x=y$. This means that the operator $(\lambda I-T)$ is surjective. On the other hand $\operatorname{Ker}(\lambda I-T)=\{0\}$ which follows from $\lambda \notin \overline{\left\{d_{i}: i \in \mathbb{N}\right\}}$. Therefore, as $\lambda_{p}(A)$ is a Fréchet space, $\lambda \in \rho(T)$; that is, $\mathbb{T} \backslash\{1\} \subseteq \rho(T)$. Since $1 \in \Gamma(T)$, it follows that $\Gamma(T)=\{1\}$.

Concerning the example in Proposition 17 we note that (i) of Theorem 10 holds but (iii) of Theorem 10 fails (as $\Gamma(T)=$ $\{1\}$ implies that $\sigma(T) \nsubseteq \mathbb{D}$ ). Moreover, (i) of Theorem 15 holds (as $\tau_{b}-\lim _{n \rightarrow \infty} T^{n}=0$ ) but (ii) and (iii) of Theorem 15 fail (because $(I-T)^{m}\left(\lambda_{p}(A)\right)$ is not closed in $\lambda_{p}(A)$ for every $m \epsilon$ $\mathbb{N})$. Of course, $\lambda_{p}(A)$ is not a prequojection.

A well-known result of Katznelson and Tzafriri states that a power bounded operator $T$ on a Banach space satisfies $\lim _{n \rightarrow \infty}\left\|T^{n+1}-T^{n}\right\|_{\text {op }}=0$ if and only if $\Gamma(T) \subseteq\{1\},[7$, Theorem 1 and p. 317 Remark]. In order to extend this result to prequojection Fréchet spaces (see Theorem 20 below) we require the following notion.

Let $X$ be a Fréchet space and $T \in \mathscr{L}(X)$. A fundamental, increasing sequence $\left\{q_{j}\right\}_{j=1}^{\infty} \subseteq \Gamma_{X}$ which generates the lctopology of $X$ is called $T$ contractively admissible if, for each $j \in \mathbb{N}$, we have

$$
q_{j}(T x) \leq q_{j}(x), \quad x \in X .
$$

Lemma 18. Let $X$ be a Fréchet space and $T \in \mathscr{L}(X)$. Then there exists a $T$ contractively admissible sequence of seminorms which generates the lc-topology of $X$ if and only if $T$ is power bounded.

Proof. If $\left\{q_{j}\right\}_{j=1}^{\infty} \subseteq \Gamma_{X}$ is $T$ contractively admissible, then it is clear from (36) that $q_{j}\left(T^{n} x\right) \leq q_{j}(x)$, for $x \in X$ and every $n \epsilon$ $\mathbb{N}_{0}, j \in \mathbb{N}$. This means precisely that $\left\{T^{n}\right\}_{n=1}^{\infty}$ is equicontinuous in $\mathscr{L}(X)$; that is, $T$ is power bounded.

Conversely, suppose that $T$ is power bounded. Let $\left\{r_{j}\right\}_{j=1}^{\infty}$ be a fundamental, increasing sequence in $\Gamma_{X}$ which generates the lc-topology of $X$. Via the equicontinuity of $\left\{T^{n}\right\}_{n=1}^{\infty}$ for every $j \in \mathbb{N}$ there exist $k(j) \geq j$ and $\alpha_{j}>0$ such that

$$
r_{j}\left(T^{n} x\right) \leq \alpha_{j} r_{k(j)}(x), \quad x \in X, n \in \mathbb{N} .
$$


Define $q_{j}(x):=\sup _{n \in \mathbb{N}_{0}} r_{j}\left(T^{n} x\right)$, for $x \in X$ and each $j \in \mathbb{N}$. Then the previous inequality implies that

$$
r_{j}(x) \leq q_{j}(x) \leq \alpha_{j} r_{k(j)}(x), \quad x \in X, j \in \mathbb{N},
$$

and so $\left\{q_{j}\right\}_{j=1}^{\infty} \subseteq \Gamma_{X}$ is a fundamental, increasing sequence determining the lc topology of $X$, which clearly satisfies (36). That is, $\left\{q_{j}\right\}_{j=1}^{\infty}$ is $T$ contractively admissible.

Remark 19. (i) For a Banach space $X$, Lemma 18 simply states that $T$ is power bounded if and only if it is a contraction for some equivalent norm in $X$.

(ii) Let $X$ be a Fréchet space and let $T \in \mathscr{L}(X)$ be an isomorphism which is bipower bounded; that is, $\left\{T^{n}: n \in \mathbb{Z}\right\}$ is equicontinuous in $\mathscr{L}(X)$. An examination of the proof of Lemma 18 shows that there exists a sequence $\left\{q_{j}\right\}_{j=1}^{\infty} \subseteq \Gamma_{X}$, again called $T$ contractively admissible, which generates the lc-topology of $X$ and satisfies, for each $j \in \mathbb{N}$,

$$
q_{j}\left(T^{n} x\right) \leq q_{j}(x), \quad x \in X, n \in \mathbb{Z} .
$$

Theorem 20. Let $X$ be a prequojection Fréchet space and let $T \in \mathscr{L}(X)$ be power bounded. The following assertions are equivalent.

(i) $\tau_{b}-\lim _{n \rightarrow \infty}\left(T^{n+1}-T^{n}\right)=0$.

(ii) $\Gamma(T) \subseteq\{1\}$ and there exists a $T$ contractively admissible sequence $\left\{p_{j}\right\}_{j=1}^{\infty} \subseteq \Gamma_{X}$ such that, for each $\lambda \in \mathbb{T} \backslash\{1\}$ and $j \in \mathbb{N}$, there exists $M_{\lambda, j}>0$ satisfying

$$
p_{j}(R(\lambda, T) x) \leq M_{\lambda, j} p_{j}(x), \quad x \in X
$$

Remark 21. (i) If $\Gamma(T) \subseteq\{1\}$, then necessarily $\mathbb{T} \backslash\{1\} \subseteq \rho(T)$ and so the resolvent family $\{R(\lambda, T): \lambda \in \mathbb{T} \backslash\{1\}\}$ is defined.

(ii) If $\Gamma(T)=\emptyset$, then (i) of Theorem 20 follows without any further conditions. Indeed, by Remark 9 we actually have $\sigma(T) \subseteq \mathbb{D}$. Then Theorem 10 implies that $\tau_{b}-\lim _{n \rightarrow \infty} T^{n}=0$ and, hence, also $\tau_{b}-\lim _{n \rightarrow \infty}\left(T^{n+1}-T^{n}\right)=0$.

(iii) If $X$ is a Banach space and $\|\cdot\|$ is any norm in $X$ for which $T$ is a contraction (i.e., $\|\cdot\|$ is $T$ contractively admissible), then the requirement (40) automatically holds with $M_{\lambda}:=\|R(\lambda, T)\|_{\mathrm{op}}$. That is, condition (ii) in Theorem 20 simply reduces to $\Gamma(T) \subseteq\{1\}$ and we recover the result of Katznelson and Tzafriri.

Proof of Theorem $20(i) \Rightarrow($ ii). As usual we distinguish two cases.

Case (I) ( $X$ is a quojection). According to Lemma 18 there is a $T$ contractively admissible sequence $\left\{q_{j}\right\}_{j=1}^{\infty} \subseteq \Gamma_{X}$ satisfying (36) and, hence, also $q_{j}\left(T^{n} x\right) \leq q_{j}(x)$, for $x \in X$ and all $j, n \in$ $\mathbb{N}$. We proceed as in the proof of Proposition 8 (now using (36) in place of (21) so that (24) becomes $\widetilde{q}_{j}\left(T_{j} \widehat{x}\right) \leq \widetilde{q}_{j}(\widehat{x})$, for $\widehat{x} \in X_{j}$ and $\left.j \in \mathbb{N}\right)$ to obtain that $X=\operatorname{proj}_{j}\left(X_{j}, Q_{j, j+1}\right)$ in such a way that, for every $j \in \mathbb{N}$, there exists a contraction $T_{j} \in$ $\mathscr{L}\left(X_{j}\right)$ satisfying $T_{j} Q_{j}=Q_{j} T$. Then also $T_{j}^{n} Q_{j}=Q_{j} T^{n}$ for all $j, n \in \mathbb{N}$. For each $j \in \mathbb{N}$, define $p_{j}(x):=\widetilde{q}_{j}\left(Q_{j} x\right)$ for $x \in X$. By the properties of projective limits $\left\{p_{j}\right\}_{j=1}^{\infty} \subseteq \Gamma_{X}$ is a fundamental sequence generating the lc-topology of $X$. Moreover,

$$
\begin{aligned}
p_{j}(T x) & =\widetilde{q}_{j}\left(Q_{j} T x\right) \\
& =\widetilde{q}_{j}\left(T_{j} Q_{j} x\right) \leq \widetilde{q}_{j}\left(Q_{j} x\right)=p_{j}(x), \quad x \in X,
\end{aligned}
$$

shows that $\left\{q_{j}\right\}_{j=1}^{\infty}$ is also $T$ contractively admissible. According to Lemma 6 (applied to the norms \|\|$_{j}:=\widetilde{q}_{j}$ and with $S_{n}:=\left(T^{n+1}-T^{n}\right), n \in \mathbb{N}$, and $S_{n}^{(j)}:=\left(T_{j}^{n+1}-T_{j}^{n}\right)$, for $j$, $n \in \mathbb{N})$, the assumption $\tau_{b}-\lim _{n \rightarrow \infty}\left(T^{n+1}-T^{n}\right)=0$ implies that $\lim _{n \rightarrow \infty}\left\|T_{j}^{n+1}-T_{j}^{n}\right\|_{\text {op }}=0$, for each $j \in \mathbb{N}$. By [7, Theorem 1] we can conclude that $\Gamma\left(T_{j}\right) \subseteq\{1\}$. On the other hand, $\sigma\left(T_{j}\right) \subseteq \overline{\mathbb{D}}$ as $T_{j}$ is a contraction and so $\sigma\left(T_{j}\right) \subseteq \mathbb{D} \cup\{1\}$; that is, $\rho\left(T_{j}\right) \supseteq \mathbb{C} \backslash(\mathbb{D} \cup\{1\})$, for $j \in \mathbb{N}$. According to Lemma 5 also $\rho(T) \supseteq \mathbb{C} \backslash(\mathbb{D} \cup\{1\})$; that is, $\Gamma(T) \subseteq\{1\}$.

Concerning (40), fix $\lambda \in \mathbb{T} \backslash\{1\}$ and $j \in \mathbb{N}$. By the previous paragraph $\lambda \in \rho(T) \cap \rho\left(T_{j}\right)$. It follows from $T_{j} Q_{j}=Q_{j} T$ that $Q_{j} R(\lambda, T)=R\left(\lambda, T_{j}\right) Q_{j}$. Hence, for $x \in X$, we have

$$
\begin{aligned}
p_{j}(R(\lambda, T) x) & =\widetilde{q}_{j}\left(Q_{j} R(\lambda, T) x\right) \\
& =\tilde{q}_{j}\left(R\left(\lambda, T_{j}\right) Q_{j} x\right) \leq\left\|R\left(\lambda, T_{j}\right)\right\|_{\mathrm{op}} \tilde{q}_{j}\left(Q_{j} x\right) \\
& =\left\|R\left(\lambda, T_{j}\right)\right\|_{\mathrm{op}} p_{j}(x)
\end{aligned}
$$

which establishes (40).

Case (II) ( $X$ is a prequojection). As noted before, $X$ and $X_{\beta}^{\prime}$ are barrelled (hence, quasi-barrelled) with $T^{\prime} \in \mathscr{L}\left(X_{\beta}^{\prime}\right)$ and $T^{\prime \prime} \in \mathscr{L}\left(X^{\prime \prime}\right)$. So, the assumption $\tau_{b}-\lim _{n \rightarrow \infty}\left(T^{n+1}-T^{n}\right)=0$ implies that $\tau_{b}-\lim _{n \rightarrow \infty}\left(\left(T^{\prime \prime}\right)^{n+1}-\left(T^{\prime \prime}\right)^{n}\right)=0$. Moreover, $X^{\prime \prime}$ is a quojection Fréchet space and $T^{\prime \prime}$ is power bounded; see Lemma 2. So, the result of Case (I) yields $\Gamma\left(T^{\prime \prime}\right) \subseteq\{1\}$. But, $\Gamma(T)=\Gamma\left(T^{\prime \prime}\right)$ (see Corollary 4$)$ and so $\Gamma(T) \subseteq\{1\}$.

By (i) $\Rightarrow$ (ii) for quojections there exists a $T^{\prime \prime}$ contractively admissible sequence $\left\{p_{j}^{\prime \prime}\right\}_{j=1}^{\infty} \subseteq \Gamma_{X}^{\prime \prime}$ such that, for every $\lambda \epsilon$ $\mathbb{V} \backslash\{1\}$ and $j \in \mathbb{N}$, there exists $M_{\lambda, j}>0$ satisfying

$$
p_{j}^{\prime \prime}\left(R\left(\lambda, T^{\prime \prime}\right) x^{\prime \prime}\right) \leq M_{\lambda, j} p_{j}^{\prime \prime}\left(x^{\prime \prime}\right), \quad x^{\prime \prime} \in X^{\prime \prime} .
$$

By Lemma 1 and Corollary 4 the seminorms $p_{j}:=p_{j}^{\prime \prime} \circ \Phi$, $j \in \mathbb{N}$, satisfy (40).

(ii) $\Rightarrow$ (i). Case (I). ( $X$ is a quojection).

Let $\left\{p_{j}\right\}_{j=1}^{\infty} \subseteq \Gamma_{X}$ be as in the statement of (ii), in which case (36) holds. Proceed as in Case (I) of the proof of (i) $\Rightarrow$ (ii) to obtain that $X=\operatorname{proj}_{j}\left(X_{j}, Q_{j, j+1}\right)$ in such a way that, for every $j \in \mathbb{N}$, there exists a contraction $T_{j} \in \mathscr{L}\left(X_{j}\right)$, satisfying $T_{j} Q_{j}=Q_{j} T$.

Claim 1. $\Gamma\left(T_{j}\right) \subseteq\{1\}$, for every $j \in \mathbb{N}$.

To establish this, let $\lambda \in \mathbb{T} \backslash\{1\}$. Since $\Gamma(T) \subseteq\{1\}$, it follows that $\lambda \in \rho(T)$, and hence, $\lambda I-T$ is surjective. But, also $Q_{j}$ : $X \rightarrow X_{j}$ is surjective. It is then routine to check from the identity $\left(\lambda I_{j}-T_{j}\right) Q_{j}=Q_{j}(\lambda I-T)$ that $\lambda I_{j}-T_{j}$ is surjective. To verify that $\lambda I_{j}-T_{j}$ is injective suppose that $\left(\lambda I_{j}-T_{j}\right) y=0$ for some $y \in X_{j}$, in which case $y=Q_{j} x$ for some $x \in X$. Accordingly,

$$
Q_{j}(\lambda I-T) x=\left(\lambda I_{j}-T_{j}\right) Q_{j} x=\left(\lambda I_{j}-T_{j}\right) y=0
$$


shows that $(\lambda I-T) x \in \operatorname{Ker} Q_{j}=\operatorname{Ker} p_{j}$. It then follows from (40) that $x=R(\lambda, T)(\lambda I-T) x \in \operatorname{Ker} p_{j}$; that is, $Q_{j} x=0$. Since $y=Q_{j} x$, we have $y=0$. Hence, $\lambda I_{j}-T_{j}$ is injective. This establishes that $\lambda \in \rho\left(T_{j}\right)$, and hence, Claim 1 follows as $\lambda \epsilon$ $\mathbb{T} \backslash\{1\}$ was arbitrary.

Fix $j \in \mathbb{N}$. From Claim 1 and the fact that $T_{j}$ is a contraction, it follows from [7, Theorem 1] that $\lim _{n \rightarrow \infty}\left\|T_{j}^{n}-T_{j}^{n+1}\right\|_{\text {op }}=0$. According to Lemma 6 (with $\left.S_{n}:=\left(T^{n+1}-T^{n}\right), n \in \mathbb{N}\right)$ we can conclude that $\tau_{b^{-}}$ $\lim _{n \rightarrow \infty}\left(T^{n+1}-T^{n}\right)=0$.

Case (II) ( $X$ is a prequojection). By Corollary 4 we have from $\Gamma(T) \subseteq\{1\}$ that $\Gamma\left(T^{\prime \prime}\right) \subseteq\{1\}$. Moreover, Lemma 2 implies that $T^{\prime \prime} \in \mathscr{L}\left(X^{\prime \prime}\right)$ is power bounded.

Let $\left\{p_{j}\right\}_{j=1}^{\infty} \subseteq \Gamma_{X}$ be as stated in part (ii). Apply Lemma 1 to construct the seminorms $\left\{p_{j}^{\prime \prime}\right\}_{j=1}^{\infty} \subseteq \Gamma_{X}^{\prime \prime}$ given there. We first verify that $\left\{p_{j}^{\prime \prime}\right\}_{j=1}^{\infty} \subseteq \Gamma_{X}^{\prime \prime}$ is $T^{\prime \prime}$ contractively admissible. Since $\left\{p_{j}\right\}_{j=1}^{\infty}$ is $T$ contractively admissible, we have $T\left(\mathcal{U}_{j}\right) \subseteq \mathcal{U}_{j}$ with $\mathcal{U}_{j}$ the closed unit ball of $p_{j}$; that is, $\mathcal{U}_{j}=p_{j}^{-1}([0,1])$, for $j \in \mathbb{N}$. By the Bi-polar Theorem, [10, Theorem 22.13] applied twice we have

$$
T^{\prime \prime}\left(\mathcal{U}_{j}^{\circ \circ}\right)=T^{\prime \prime}\left(\overline{\mathcal{U}}_{j}^{\sigma}\right) \subseteq{\overline{T\left(\mathcal{U}_{j}\right)}}^{\sigma} \subseteq \overline{\mathcal{U}}_{j}^{\sigma}=\mathscr{U}_{j}^{\circ},
$$

where $\bar{V}^{\sigma}$ denotes the closure for the weak ${ }^{*}$ topology $\sigma\left(X^{\prime \prime}\right.$, $X^{\prime}$ ) of a subset $V \subseteq X^{\prime \prime}$ (or, of $V \subseteq X \subseteq X^{\prime \prime}$ ). Then (45) implies that $p_{j}^{\prime \prime}\left(T^{\prime \prime} x^{\prime \prime}\right) \leq p_{j}^{\prime \prime}\left(x^{\prime \prime}\right)$ for each $x^{\prime \prime} \in X^{\prime \prime}$ and $j \in \mathbb{N}$; that is, $\left\{p_{j}^{\prime \prime}\right\}_{j=1}^{\infty}$ is $T^{\prime \prime}$ contractively admissible.

It follows from (40) that $R(\lambda, T)\left(\mathcal{U}_{j}\right) \subseteq \mathcal{U}_{j}$, for all $\lambda \in \mathbb{T} \backslash$ $\{1\}$ and $j \in \mathbb{N}$. Using $\left.R\left(\lambda, T^{\prime \prime}\right)\right|_{X}=R(\lambda, T)$ (c.f. Corollary 4 ) one can repeat the argument via the Bi-polar Theorem to conclude that $R\left(\lambda, T^{\prime \prime}\right)\left(\mathcal{U}_{j}^{\circ}\right) \subseteq M_{\lambda, j} \mathcal{U}_{j}^{\circ}$, which then implies that

$$
p_{j}^{\prime \prime}\left(R\left(\lambda, T^{\prime \prime}\right) x^{\prime \prime}\right) \leq M_{\lambda, j} p_{j}^{\prime \prime}\left(x^{\prime \prime}\right), \quad x^{\prime \prime} \in X^{\prime \prime} .
$$

So, the conditions in part (ii) are satisfied for the power bounded operator $T^{\prime \prime} \in \mathscr{L}\left(X^{\prime \prime}\right)$ with respect to $\left\{p_{j}^{\prime \prime}\right\}_{j=1}^{\infty}$. Applying (ii) $\Rightarrow$ (i) for the quojection Fréchet space $X^{\prime \prime}$ we conclude that $\tau_{b}-\lim _{n \rightarrow \infty}\left(\left(T^{\prime \prime}\right)^{n+1}-\left(T^{\prime \prime}\right)^{n}\right)=0$. But, $\left.T^{\prime \prime}\right|_{X}=$ $T$ with $X$ closed in $X^{\prime \prime}$. So, $\tau_{b}-\lim _{n \rightarrow \infty}\left(T^{n+1}-T^{n}\right)=0$; that is, (i) holds.

Let $X$ be a prequojection Fréchet space and $T \in \mathscr{L}(X)$ be power bounded. By Remark 9 we have $\sigma(T) \subseteq \overline{\mathbb{D}}$. Suppose that $T$ is actually bipower bounded. Then also $\sigma\left(T^{-1}\right) \subseteq \overline{\mathbb{D}}$. Clearly, $0 \in \rho(T)$. Moreover, if $\mu \in \mathbb{D} \backslash\{0\}$, then $1 / \mu \in \mathbb{C} \backslash \overline{\mathbb{D}}$ and so $1 / \mu \in \rho\left(T^{-1}\right)$, that is, $\left((1 / \mu) I-T^{-1}\right)^{-1} \in \mathscr{L}(X)$. It is routine to check that $R_{\mu}:=-(1 / \mu) T^{-1}\left((1 / \mu) I-T^{-1}\right)^{-1} \epsilon$ $\mathscr{L}(X)$ satisfies $(\mu I-T) R_{\mu}=I=R_{\mu}(\mu I-T)$ and hence, $(\mu I-T)$ is invertible in $\mathscr{L}(X)$ with $(\mu I-T)^{-1}=R_{\mu}$. This shows that $\mathbb{D} \subseteq \rho(T)$. Accordingly, $\sigma(T) \subseteq \mathbb{T}$; for $X$ a Banach space, see [31, Proposition 1.31], for example. Suppose now, in addition, that $\sigma(T)=\{1\}$ in which case $\sigma(T-I)=\{0\}$; that is, $T$ is quasinilpotent. For $X$ a Banach space, a classical result of Gelfand-Hille then states that necessarily $T=I$; see the survey article [32] for a complete discussion of this topic. The following fact is an extension of this result.
Corollary 22. Let $X$ be a prequojection Fréchet space and $T \epsilon$ $\mathscr{L}(X)$ be an isomorphism which is bipower bounded. Suppose that $\Gamma(T)=\{1\}$ and there exists a $T$ contractively admissible sequence $\left\{p_{j}\right\}_{j=1}^{\infty} \subseteq \Gamma_{X}$ such that, for each $\lambda \in \mathbb{T} \backslash\{1\}$, the inequalities (40) are satisfied. Then $T=I$.

Proof. According to Theorem 20 we can conclude that $\tau_{b^{-}}$ $\lim _{n \rightarrow \infty}\left(T^{n+1}-T^{n}\right)=0$. Fix $x \in X$. For each $j \in \mathbb{N}$, it follows that

$$
\begin{aligned}
p_{j}((T-I) x) & =p_{j}\left(T^{-n} T^{n}(T-I) x\right) \\
& \leq p_{j}\left(T^{n}(T-I) x\right)=p_{j}\left(\left(T^{n+1}-T^{n}\right) x\right)
\end{aligned}
$$

for every $n \in \mathbb{N}$. Since $\lim _{n \rightarrow \infty}\left(T^{n+1}-T^{n}\right) x=0$, it follows that $p_{j}((T-I) x)=0$ with $j \in \mathbb{N}$ arbitrary; that is, $T x=x$. So, $T=I$.

\section{Operator Ideals and Uniform Mean Ergodicity}

Let $X, Y$ be lcHs'. An operator $T \in \mathscr{L}(X, Y)$ is called Montel (resp. reflexive) if $T$ maps bounded subsets of $X$ into relatively compact (resp. relatively weakly compact) subsets of $Y$ [33] (resp., [34]). According to Grothendieck, [35, Chapter 5, Part 2], $T$ is called compact (resp., weakly compact) if there exists a 0 -neighbourhood $\mathcal{U} \subseteq X$ such that $T(\mathcal{U})$ is relatively compact (resp., relatively weakly compact) in $Y$. Clearly, the 2-sided ideal $\mathscr{M}(X, Y)$ (resp., $\mathscr{R}(X, Y)$ ) of all Montel (resp., reflexive) operators coincides with the 2 -sided ideal $\mathscr{K}(X, Y)$ (resp., $\mathscr{W} \mathscr{K}(X, Y)$ ) of all compact (resp., weakly compact) operators whenever $X, Y$ are Banach spaces. For general lcHs' we always have $\mathscr{K}(X, Y) \subseteq \mathscr{M}(X, Y)$ but the containment may be proper; consider the identity operator on an infinite dimensional Montel lcHs. Clearly, $\mathscr{M}(X, Y) \subseteq \mathscr{R}(X, Y)$ and $\mathscr{W} \mathscr{K}(X, Y) \subseteq \mathscr{K}(X, Y)$. Criteria for membership of $\mathscr{M}(X, Y)$ (resp. $\mathscr{R}(X, Y)$ ) occur in Theorem 9.2.1 (resp. Corollary 9.3.2) of [36], for example.

In this section we present various connections between the uniform convergence of sequences of operators generated by an operator $T \in \mathscr{H}(X)$ and the uniform mean ergodicity of $T$, where $\mathscr{H}$ stands for one of the operator ideals $\mathscr{K}, \mathscr{M}$, $\mathscr{W} \mathscr{K}, \mathscr{R}$.

Every compact operator $T$ acting in a Banach space has the property that $(I-T)$ has closed range. Hence, if $\lim _{n \rightarrow \infty}\left(\left\|T^{n}\right\|_{\text {op }} / n\right)=0$, then $T$ is uniformly mean ergodic [1, p. 711, Corollary 4], [4, p. 87, Theorem 2.1]. For any lcHs $X$ and $T \in \mathscr{K}(X)$, it is also the case that $(I-T)(X)$ is a closed subspace of $X$ [36, Theorem 9.10.1]. Hence, if $X$ is a prequojection Fréchet space, then Theorem 3.5 of [6] implies that $T$ is uniformly mean ergodic whenever $\tau_{b^{-}}$ $\lim _{n \rightarrow \infty}\left(T^{n} / n\right)=0$ (equivalently, $\tau_{s}-\lim _{n \rightarrow \infty}\left(T^{n} / n\right)=0$ because $K \in \mathscr{K}(X)$; see Remark 26(ii)). Since $\mathscr{K}(X) \subseteq$ $\mathscr{M}(X)$, the question arises of whether the same is true for $T \in \mathscr{M}(X)$ ? This is indeed so; see Theorem 27 below.

In a lcHs $X$ all relatively $\sigma\left(X, X^{\prime}\right)$-compact sets and all relatively sequentially $\sigma\left(X, X^{\prime}\right)$-compact sets are necessarily relatively countably $\sigma\left(X, X^{\prime}\right)$ compact. These are the only implications between these three notions which hold in general. 
All three notions coincide whenever $X_{\sigma}$ is angelic [37, p. 31]. Such spaces $X$ include all Fréchet spaces (actually, all (LF)spaces), all (DF)-spaces and many more, [37, Section 3.10], [38, Theorem 11, Examples 1.2].

Operators $T \in \mathscr{L}(X)$ for which $\left\{T_{[n]}\right\}_{n=1}^{\infty} \subseteq \mathscr{L}(X)$ is equicontinuous will be called Cesàro bounded; see [4, p. 72] for $X$ a Banach space.

Proposition 23. Let $X$ be a lcHs such that $X_{\sigma}$ is angelic and $T \in \mathscr{L}(X)$.

(i) If $T \in \mathscr{R}(X)$ is Cesàro bounded and satisfies $\tau_{s^{-}}$ $\lim _{n \rightarrow \infty}\left(T^{n} / n\right)=0$, then $T$ is mean ergodic.

(ii) If $T \in \mathscr{M}(X)$ is Cesàro bounded and satisfies $\tau_{b^{-}}$ $\lim _{n \rightarrow \infty}\left(T^{n} / n\right)=0$, then $T$ is uniformly mean ergodic.

Proof. (i) Fix $x \in X$. It follows from (13) that

$$
\begin{aligned}
T_{[n]} x & =T_{[n]}(I-T) x+T_{[n]} T x \\
& =\frac{1}{n}\left(T-T^{n+1}\right) x+T T_{[n]} x, \quad n \in \mathbb{N} .
\end{aligned}
$$

The equicontinuity of $\left\{T_{[n]}\right\}_{n=1}^{\infty}$ ensures that $\left\{T_{[n]} x\right\}_{n=1}^{\infty} \in$ $\mathscr{B}(X)$. Since $T \in \mathscr{R}(X)$, the set $\left\{T\left(T_{[n]} x\right)\right\}_{n=1}^{\infty}$ is relatively weakly compact in $X$. Moreover, $\lim _{n \rightarrow \infty}(1 / n)\left(T-T^{n+1}\right) x=$ 0 in $X$ because of $\tau_{s}-\lim _{n \rightarrow \infty}\left(T^{n} / n\right)=0$. These facts, together with $X_{\sigma}$ being angelic and (48), show that $\left\{T_{[n]} x\right\}_{n=1}^{\infty}$ is relatively weakly (hence, relatively weakly sequentially) compact in $X$. Since $x$ is arbitrary, we can apply Theorem 2.4 of [39] (an examination of its proof shows that it is not necessary to assume the barrelledness of $X$ stated there because of the equicontinuity of $\left\{T_{[n]}\right\}_{n=1}^{\infty}$ assumed here) to conclude that $T$ is mean ergodic.

(ii) By part (i) the operator $T$ is mean ergodic, that is, $\tau_{s^{-}}$ $\lim _{n \rightarrow \infty} T_{[n]}=: P$ exists in $\mathscr{L}_{s}(X)$. In particular, $P=T P=P T$ (which follows from (13)) and so $P=T_{[n]} P=P T_{[n]}$, for $n \in \mathbb{N}$.

To establish the uniform mean ergodicity of $T$, fix $p \in \Gamma_{X}$, $\varepsilon>0$, and $B \in \mathscr{B}(X)$. By the equicontinuity of $\left\{T_{[n]}\right\}_{n=1}^{\infty}$ there exist $M>0$ and $q \in \Gamma_{X}$ such that

$$
p\left(\left(T_{[n]}-P\right) x\right) \leq M q(x), \quad x \in X, n \in \mathbb{N} .
$$

On the other hand, $T(B)$ is a relatively compact subset of $X$ and so there exist $z_{1}, \ldots, z_{h} \in T(B)$ such that, for every $y \in$ $T(B)$, we have $q\left(y-z_{i}\right)<\varepsilon /(2 M)$ for some $i \in\{1, \ldots, h\}$. Hence, via (49) we obtain, for every $x \in B$ and $n \in \mathbb{N}$, that

$$
\begin{aligned}
p\left(T_{[n]} T x-P x\right) & =p\left(\left(T_{[n]}-P\right) T x\right) \\
& \leq p\left(\left(T_{[n]}-P\right)\left(T x-z_{i}\right)\right)+p\left(\left(T_{[n]}-P\right) z_{i}\right) \\
& \leq M q\left(T x-z_{i}\right)+p\left(\left(T_{[n]}-P\right) z_{i}\right) \\
& <\frac{\varepsilon}{2}+p\left(\left(T_{[n]}-P\right) z_{i}\right) .
\end{aligned}
$$

It follows that

$$
\sup _{x \in B} p\left(T_{[n]} T x-P x\right) \leq \frac{\varepsilon}{2}+\max _{i=1, \ldots, h} p\left(\left(T_{[n]}-P\right) z_{i}\right), \quad n \in \mathbb{N},
$$

with $\lim _{n \rightarrow \infty} \max _{i=1, \ldots, h} p\left(\left(T_{[n]}-P\right) z_{i}\right)=0$. The arbitrariness of $\varepsilon>0$ implies that $\lim _{n \rightarrow \infty} \sup _{x \in B} p\left(T_{[n]} T x-P x\right)=0$. So, $\tau_{b}-\lim _{n \rightarrow \infty} T_{[n]} T=P$.

Finally, the arbitrariness of $p \in \Gamma_{X}$ and of $B \in \mathscr{B}(X)$ together with the assumption $\tau_{b}-\lim _{n \rightarrow \infty}\left(T^{n} / n\right)=0$ implies, via (48), that $T$ is uniformly mean ergodic.

Remark 24. (i) Let $X$ be a lcHs and let $T \in \mathscr{L}(X)$ be mean ergodic with $P:=\tau_{s}-\lim _{n \rightarrow \infty} T_{[n]}$. Then it follows from $P=$ $P T$ that $P \in \mathscr{H}(X)$ whenever $T \in \mathscr{H}(X)$ (here, $\mathscr{H}$ stands for the operator ideal $\mathscr{K}, \mathscr{M}, \mathscr{W} \mathscr{K}$, or $\mathscr{R})$. In particular, if $T \in$ $\mathscr{K}(X)$, then $\operatorname{Fix}(T):=\{x \in X: T x=x\}=\operatorname{Ker}(I-T)=P(X)$ is finite dimensional, [36, Theorem 9.10.1(1)].

(ii) Let $X$ be a lcHs such that $X_{\sigma}$ is angelic. Then the class of all weakly completely continuous operators in $\mathscr{L}(X)$ in the sense of Definition 2 in [40] is precisely $\mathscr{W} \mathscr{K}(X)$. Moreover, if $X$ is additionally barrelled, then, for any $T \in$ $\mathscr{L}(X)$, the boundedness of the set $\left\{T^{n}\right\}_{n=1}^{\infty}$ in $\mathscr{L}_{s}(X)$ is equivalent to $T$ being power bounded. In particular, $T$ is necessarily Cesàro bounded and satisfies $\tau_{s}-\lim _{n \rightarrow \infty}\left(T^{n} / n\right)=$ 0 . Accordingly, the containment $\mathscr{W} \mathscr{K}(X) \subseteq \mathscr{R}(X)$ shows that Proposition 23(i) is an extension of the following result of Altman [40, Theorem].

Fact 1. Let $X$ be a barrelled lcHs with $X_{\sigma}$ being angelic. Then every power bounded operator $T \in \mathscr{W} \mathscr{K}(X)$ is mean ergodic.

The following technical result should be compared with [33, Proposition 3.1].

Lemma 25. Let $X$ be a quojection Fréchet space, and let $Y$ be a Fréchet space and $T \in \mathscr{M}(X, Y)$ (resp. $T \in \mathscr{R}(X, Y)$ ). Suppose that $X=\operatorname{proj}_{j}\left(X_{j}, Q_{j, j+1}\right)$, with $X_{j}$ a Banach space (having norm \|\|$\left._{j}\right)$ and surjective linking maps $Q_{j, j+1} \in \mathscr{L}\left(X_{j+1}, X_{j}\right)$, for all $j \in \mathbb{N}$, and that $Y=\operatorname{proj}_{j}\left(Y_{j}, R_{j, j+1}\right)$, with $Y_{j}$ a Banach space (having norm ||||||$_{j}$ ) and linking maps $R_{j, j+1} \in$ $\mathscr{L}\left(Y_{j+1}, Y_{j}\right)$ for all $j \in \mathbb{N}$. Then, for every $j \in \mathbb{N}$, there exist $k(j) \geq j$ and $T_{j} \in \mathscr{K}\left(X_{k(j)}, Y_{j}\right)\left(\right.$ resp. $\left.T_{j} \in \mathscr{W} \mathscr{K}\left(X_{k(j)}, Y_{j}\right)\right)$ such that

$$
R_{j} T=T_{j} Q_{k(j)},
$$

where $R_{j} \in \mathscr{L}\left(Y, Y_{j}\right), j \in \mathbb{N}$, is the canonical projection of $Y$ into $Y_{j}$ (i.e., $R_{j, j+1} \circ R_{j+1}=R_{j}$ ).

Proof. If we define $q_{j}(x):=\left\|Q_{j} x\right\|_{j}$ for $x \in X$ and $j \in \mathbb{N}$ and $r_{j}(y):=\|\| R_{j} y \mid \|_{j}$ for $y \in Y$ and $j \in \mathbb{N}$, then $\left\{q_{j}\right\}_{j=1}^{\infty}$ and $\left\{r_{j}\right\}_{j=1}^{\infty}$ are fundamental sequences of seminorms generating the lc-topology of $X$ and of $Y$, respectively.

Fix $j \in \mathbb{N}$. The continuity of $T$ implies that there exist $k(j) \geq j$ and $C_{j}>0$ satisfying

$$
r_{j}(T x) \leq C_{j} q_{k(j)}(x), \quad x \in X,
$$

or equivalently, that

$$
\left\|R_{j} T_{x} \mid\right\|_{j} \leq C_{j}\left\|Q_{k(j)} x\right\|_{k(j)}, \quad x \in X .
$$

As noted before such an inequality ensures that there exists $T_{j} \in \mathscr{L}\left(X_{k(j)}, Y_{j}\right)$ defined via $R_{j} T=T_{j} Q_{k(j)}$. 
Denote by $\mathscr{U}_{k(j)}$ the closed unit ball of $X_{k(j)}$. Since $X$ is a quojection Fréchet space, there exists $B \in \mathscr{B}(X)$ such that $\mathcal{U}_{k(j)} \subseteq Q_{k(j)}(B)$ [17, Proposition 1]. Since $T$ is Montel (resp. reflexive) and $R_{j}$ is continuous, it follows from $T_{j}\left(\mathscr{U}_{k(j)}\right) \subseteq$ $T_{j}\left(Q_{k(j)}(B)\right)=R_{j}(T(B))$, with $R_{j}(T(B))$ a relatively compact subset (resp. relatively weakly compact subset) of $Y_{j}$, that $T_{j}\left(\mathcal{U}_{k(j)}\right)$ is a relatively compact (resp. relatively weakly compact) subset of $Y_{j}$. That is, $T_{j} \in \mathscr{K}\left(X_{k(j)}, Y_{j}\right)$ (resp. $T_{j} \in$ $\left.\mathscr{W} \mathscr{K}\left(X_{k(j)}, Y_{j}\right)\right)$.

Remark 26. (i) Let $X=\operatorname{proj}_{j}\left(X_{j}, Q_{j, j+1}\right)$ be a quojection Fréchet space and $T \in \mathscr{L}(X)$. Suppose, for every $j \in \mathbb{N}$, that there exists $C_{j}>0$ such that $q_{j}(T x) \leq C_{j} q_{j}(x)$ for $x \in X$ (here, the notation is according to Lemma 25 and its proof with $Y:=X)$. Then, for every $j \in \mathbb{N}$, there exists $T_{j} \in \mathscr{L}\left(X_{j}\right)$ satisfying $Q_{j} T=T_{j} Q_{j}$. So, if $T \in \mathscr{M}(X)$ (resp., $\left.T \in \mathscr{R}(X)\right)$, then each $T_{j} \in \mathscr{K}\left(X_{j}\right)$ (resp., $T_{j} \in \mathscr{W} \mathscr{K}\left(X_{j}\right)$ ).

(ii) Let $X$ be a Fréchet space and $T \in \mathscr{M}(X)$. Then $\tau_{s^{-}}$ $\lim _{n \rightarrow \infty}\left(T^{n} / n\right)=0$ if and only if $\tau_{b}-\lim _{n \rightarrow \infty}\left(T^{n} / n\right)=0$.

As $\tau_{s} \subseteq \tau_{b}$, it suffices to show $\tau_{s}-\lim _{n \rightarrow \infty}\left(T^{n} / n\right)=0$ implies $\tau_{b}-\lim _{n \rightarrow \infty}\left(T^{n} / n\right)=0$.

Since $X$ is a Fréchet space and $\tau_{s}-\lim _{n \rightarrow \infty}\left(T^{n} / n\right)=0$, the set $\left\{T^{n} / n\right\}_{n=1}^{\infty}$ is equicontinuous in $\mathscr{L}(X)$; that is, for every $p \in$ $\Gamma_{X}$ there exist $q \in \Gamma_{X}$ and $M>0$ such that

$$
p\left(\frac{T^{n} x}{n}\right) \leq M q(x), \quad x \in X, n \in \mathbb{N} .
$$

Now, fix $p \in \Gamma_{X}, B \in \mathscr{B}(X)$, and $\varepsilon>0$. Choose $q \in \Gamma_{X}$ and $M>0$ according to (55). Since $T$ is a Montel operator, $T(B)$ is a relatively compact subset of $X$ and so there exist $x_{1}, \ldots, x_{k} \in X$ such that

$$
T(B) \subseteq \bigcup_{i=1}^{k}\left(x_{i}+\frac{\varepsilon}{2 M} \mathcal{U}_{q}\right),
$$

with $\mathscr{U}_{q}:=\{x \in X: q(x) \leq 1\}$. Let $x \in B$. By (56) there exist $i \in\{1, \ldots, k\}$ and $z \in \mathcal{U}_{q}$ such that $T(x)=x_{i}+(\varepsilon / 2 M) z$. Then, by (55), we have for every $n>1$ that

$$
\begin{aligned}
p\left(\frac{T^{n} x}{n}\right) & =p\left(\frac{T^{n-1}}{n} T(x)\right) \\
& \leq p\left(\frac{T^{n-1} x_{i}}{n}\right)+\frac{\varepsilon}{2 M} p\left(\frac{T^{n-1} z}{n}\right) \\
& \leq p\left(\frac{T^{n-1} x_{i}}{n-1}\right)+\frac{\varepsilon}{2} .
\end{aligned}
$$

But, $p\left(T^{n-1} x_{i} /(n-1)\right) \rightarrow 0$ as $n \rightarrow \infty$. So, there exists $n_{0} \in$ $\mathbb{N}$ (depending only on $x_{i}$ ) such that $p\left(T^{n} x / n\right)<\varepsilon$ for every $n \geq n_{0}$. Since $x$ is arbitrary and the set $\left\{x_{1}, \ldots, x_{k}\right\}$ is finite, we can conclude that $\sup _{x \in B} p\left(T^{n} x / n\right) \rightarrow 0$ for $n \rightarrow \infty$. By the arbitrariness of $B$ and $p$ we have $\tau_{b}-\lim _{n \rightarrow \infty}\left(T^{n} / n\right)=0$.

The following result should be compared with Proposition 23(ii). We point out (even if $\operatorname{dim}(X)<\infty$ ) that a Cesàro bounded operator $T$ need not satisfy $T^{n} / n \rightarrow 0$ in $\mathscr{L}_{s}(X)[4$, p. 85].
Theorem 27. Let $X$ be a prequojection Fréchet space and $T \in$ $\mathscr{M}(X)$. If $\tau_{s}-\lim _{n \rightarrow \infty}\left(T^{n} / n\right)=0$, then $T$ is uniformly mean ergodic.

Proof. We have the following two cases.

Case (I) ( $X$ is a quojection). The condition $\tau_{s^{-}}$ $\lim _{n \rightarrow \infty}\left(T^{n} / n\right)=0$ ensures that both $\tau_{b}-\lim _{n \rightarrow \infty}\left(T^{n} / n\right)=0$ (see Remark 26(ii)) and that we can represent $X=\operatorname{proj}_{j}\left(X_{j}\right.$, $\left.Q_{j, j+1}\right)$ such that, for every $j \in \mathbb{N}$, there exists $T_{j} \in \mathscr{L}\left(X_{j}\right)$ satisfying $Q_{j} T=T_{j} Q_{j}$; see the proof of Proposition 8 . According to Lemma 25 and Remark 26(i) we have $T_{j} \in \mathscr{K}$ $\left(X_{j}\right)$ for all $j \in \mathbb{N}$. Moreover, $T_{j}^{n} / n \rightarrow 0$ in $\mathscr{L}_{b}\left(X_{j}\right)$ for $n \rightarrow \infty$; see Remark 26(ii) and Lemma 6 with $S_{n}:=T^{n} / n$, for $n \in \mathbb{N}$.

Since $T_{j} \in \mathscr{K}\left(X_{j}\right)$ and $T_{j}^{n} / n \rightarrow 0$ in $\mathscr{L}_{b}\left(X_{j}\right)$ for $n \rightarrow$ $\infty$, for every $j \in \mathbb{N}$, each $T_{j}$ is uniformly mean ergodic $[1, \mathrm{p}$. 711 , Corollary 4], which implies that $T$ is also uniformly mean ergodic; see Lemma 7.

Case (II) ( $X$ is a prequojection). As noted before $X$ and $X_{\beta}^{\prime}$ are barrelled (hence, quasi-barrelled) with $T^{\prime} \in \mathscr{L}\left(X_{\beta}^{\prime}\right)$ and $T^{\prime \prime} \in \mathscr{L}\left(X^{\prime \prime}\right)$. So, the condition $\tau_{b}-\lim _{n \rightarrow \infty}\left(T^{n} / n\right)=0$ (see Remark 26(ii)) implies that $\tau_{b}-\lim _{n \rightarrow \infty}\left(\left(T^{\prime \prime}\right)^{n} / n\right)=0$. Moreover, $X^{\prime \prime}$ is a quojection Fréchet space. Also, Corollaries 2.3 and 2.4 of [33] yield that $T^{\prime \prime} \in \mathscr{M}\left(X^{\prime \prime}\right)$. We can then apply Case (I) to conclude that $T^{\prime \prime}$ is uniformly mean ergodic. So, $T$ is also uniformly mean ergodic as $\left.T^{\prime \prime}\right|_{X}=T$ and $X$ is a closed subspace of $X^{\prime \prime}$.

It was noted prior to Proposition 23, for $X$ a prequojection Fréchet space and $T \in \mathscr{K}(X)$, that $T$ is uniformly mean ergodic whenever $\tau_{b}-\lim _{n \rightarrow \infty}\left(T^{n} / n\right)=0$. Since $\mathscr{K}(X) \subset$ $\mathscr{M}(X)$ in general, Theorem 27 can be viewed as an extension of this fact.

Corollary 28. Let $X$ be a prequojection Fréchet space and let $T \in \mathscr{M}(X)$ be power bounded. Then $\Gamma(T) \subseteq\{1\}$ if and only if $\tau_{b}-\lim _{n \rightarrow \infty}\left(T^{n+1}-T^{n}\right)=0$.

Proof. If $\tau_{b}-\lim _{n \rightarrow \infty}\left(T^{n+1}-T^{n}\right)=0$, then Theorem 20 yields $\Gamma(T) \subseteq\{1\}$.

Conversely, suppose that $\Gamma(T) \subseteq\{1\}$. Since $T$ is power bounded, $T^{n} / n \rightarrow 0$ in $\mathscr{L}_{b}(X)$ for $n \rightarrow \infty$ and so $T$ is uniformly mean ergodic by Theorem 27. By Theorem 3.5 of [6] this is equivalent to the fact that $(I-T)(X)$ is closed in $X$. So, by Theorem 15 (ii) $\Leftrightarrow$ (iii) we can conclude that $\tau_{b^{-}}$ $\lim _{n \rightarrow \infty}\left(T^{n+1}-T^{n}\right)=0$.

In a Banach space $X$, an operator $T \in \mathscr{L}(X)$ is called quasi-compact if there exist $m \in \mathbb{N}$ and $K \in \mathscr{K}(X)$ such that $\left\|T^{m}-K\right\|_{\text {op }}<1[8, \S 6],[4$, p. 88]. For example, if some power of $T \in \mathscr{L}(X)$ is compact or if some power of $T$ has norm less than one, then $T$ is quasi-compact. For a quasi-compact operator $T$ it is known that $\tau_{s}-\lim _{n \rightarrow \infty}\left(T^{n} / n\right)=0$ suffices for $T$ to be uniformly mean ergodic [1, Ch.VIII, Corollary 8.4]. For $X$ non-normable, the question arises of how to extend the notion of a quasi-compact operator. 
According to [9, Definition 1], for a lcHs $X$ an operator $T \in \mathscr{L}(X)$ is called quasi-precompact if there exists a 0 neighbourhood $W$ such that for every 0 -neighbourhood $\mathcal{U}$ in $X$ there exist $p \in \mathbb{N}$ and a finite set $F \subseteq X$ (both depending on $\mathcal{U})$ with the property that $T^{p}(W) \subseteq \cup_{y \in F}(y+\mathcal{U})$. For $X$ a Banach space, this notion coincides precisely with $T$ being quasi-compact [9, Theorem 3]. In [41] an operator $K \in \mathscr{L}(X)$ is called $V$-compact if $K(V)$ is a relatively compact subset of $X$, where $V$ is some 0 -neighbourhood in $X$. More generally, $T \in \mathscr{L}(X)$ is called $V$-quasicompact [41, Definition 2.1], if there exist $m \in \mathbb{N}$, a $V$-compact operator $K$ and $\delta \in(0,1)$ such that $\left(T^{m}-K\right)(V) \in \mathscr{B}(X)$ and $\left(T^{m}-K\right)(V) \subseteq \delta V$.

Lemma 29. Let $X$ be a lcHs and let $V$ be any 0-neighbourhood in $X$. Then every $V$-quasicompact operator is quasi-precompact.

Proof. Let $T \in \mathscr{L}(X)$ be $V$-quasicompact. Choose $m \in \mathbb{N}$, a $V$-compact operator $K$ and $\delta \in(0,1)$ such that the set $B:=$ $\left(T^{m}-K\right)(V)$ is bounded and $B \subseteq \delta V$. Then

$$
\left(T^{m}-K\right)^{2}(V)=\left(T^{m}-K\right)(B) \subseteq\left(T^{m}-K\right)(\delta V)=\delta B .
$$

Proceeding inductively yields

$$
\left(T^{m}-K\right)^{p}(V) \subseteq \delta^{p-1} B, \quad p \in \mathbb{N} .
$$

Fix $p \in \mathbb{N}$. Note that $T^{m}$ and $K$ need not commute. By expanding $\left(T^{m}-K\right)^{p}$ it can be seen that $\left(T^{m}-K\right)^{p}=T^{m p}-$ $H_{p}$, where $H_{p}$ is a finite sum of operators all of the form $A K$ or $B K\left(T^{m}\right)^{n}$ with $A, B \in \mathscr{L}(X)$ and $n \in\{1, \ldots, p-1\}$. The claim is that $H_{p}$ is a $V$-compact operator. Indeed, since $A K$ is always $V$-compact and the finite sum of $V$-compact operators is clearly $V$-compact, it suffices to show that $K\left(T^{m}\right)^{n}$ (hence, also $\left.B K\left(T^{m}\right)^{n}\right)$ is $V$-compact for all $1 \leq n<p$.

For $n=1$, observe that $T^{m}(V)=K(V)+B \subseteq K(V)+\delta V$ yields

$$
K T^{m}(V) \subseteq K^{2}(V)+\delta K(V),
$$

which is a relatively compact subset of $X$. For $n=2$, we then have

$$
\left(T^{m}\right)^{2}(V) \subseteq T^{m}(K(V)+\delta V)=T^{m} K(V)+\delta T^{m}(V)
$$

and, hence, that

$$
K\left(T^{m}\right)^{2}(V) \subseteq K T^{m} K(V)+\delta K T^{m}(V) .
$$

Since both $T^{m} K(V)$ and $K T^{m}(V)$ are relatively compact, it follows that $K\left(T^{m}\right)^{2}(V)$ is also relatively compact. This argument can be continued to yield the above stated claim for all $1 \leq n<p$.

Define now $W:=V$ and let $\mathcal{U}$ be any convex, balanced 0 neighbourhood of $X$. Since $B$ is bounded, there is $\lambda>0$ such that $B \subseteq(1 / 2) \lambda \mathcal{U}$. Choose $\widetilde{p} \in \mathbb{N}$ large enough to ensure that $\delta^{\widetilde{p}^{-1}} \lambda \leq 1$. It follows from (59) that

$$
\left(T^{m \tilde{p}}-H_{\tilde{p}}\right)(W)=\left(T^{m}-K\right)^{\tilde{p}}(V) \subseteq \delta^{\tilde{p}-1} B
$$

and so

$$
\begin{aligned}
T^{m \tilde{p}}(W) & \subseteq H_{\tilde{p}}(V)+\left(T^{m \tilde{p}}-H_{\tilde{p}}\right)(W) \\
& \subseteq H_{\tilde{p}}(V)+\frac{1}{2} \delta^{\tilde{p}-1} \lambda \mathscr{U} \subseteq H_{\tilde{p}}(V)+\frac{1}{2} \mathscr{U} .
\end{aligned}
$$

But, $H_{\tilde{p}}(V)$ is relatively compact and so there is a finite set $F \subseteq X$ such that $H_{\tilde{p}}(V) \subseteq \cup_{x \in F}(x+(1 / 2) \mathcal{U})$. Accordingly,

$$
T^{m \tilde{p}}(W) \subseteq \frac{1}{2} \mathcal{U}+\bigcup_{x \in F}\left(x+\frac{1}{2} W\right) \subseteq \bigcup_{x \in F}(x+\mathcal{U}),
$$

which establishes that $T$ is quasi-precompact.

Returning to mean ergodicity, we have the following result of Pietsch [9, Theorem 7].

Fact 2. Let $X$ be a complete, barrelled $\mathrm{lcHs}$ and let $T \in \mathscr{L}(X)$ be a quasi-precompact operator satisfying $\tau_{s^{-}}$ $\lim _{n \rightarrow \infty}\left(T^{n} / n\right)=0$. Then $T$ is uniformly mean ergodic and $\operatorname{Fix}(T)=\operatorname{Ker}(I-T)$ is finite dimensional.

In order to be able to extend this result to a larger class of operators we recall, for a Banach space $X$, that $T \in \mathscr{L}(X)$ is quasi-compact if and only if there exists a sequence $\left\{K_{n}\right\}_{n=1}^{\infty} \subseteq$ $\mathscr{K}(X)$ such that $\lim _{n \rightarrow \infty}\left\|T^{n}-K_{n}\right\|=0[4$, p. 88, Lemma 2.4].

Definition 30. Let $X$ be a lcHs. An operator $T \in \mathscr{L}(X)$ is called quasi-Montel (resp., quasi reflexive) if there exists a sequence $\left\{M_{n}\right\}_{n=1}^{\infty} \subseteq \mathscr{M}(X)$ (resp., $\left.\left\{M_{n}\right\}_{n=1}^{\infty} \subseteq \mathscr{R}(X)\right)$ such that $\left(T^{n}-M_{n}\right) \rightarrow 0$ in $\mathscr{L}_{b}(X)$ as $n \rightarrow \infty$.

Remark 31. (i) Let $X$ be a Fréchet space and $T \in \mathscr{L}(X)$ be quasi-Montel. Then $T^{\prime \prime} \in \mathscr{L}\left(X^{\prime \prime}\right)$ is also quasi-Montel. Indeed, in the notation of Definition 30, we have $\left\{M_{n}^{\prime \prime}\right\}_{n=1}^{\infty} \subseteq$ $\mathscr{M}\left(X^{\prime \prime}\right)$ [33, Corollaries 2.3 and 2.4], with $\left(\left(T^{\prime \prime}\right)^{n}-M_{n}^{\prime \prime}\right) \rightarrow 0$ in $\mathscr{L}_{b}\left(X^{\prime \prime}\right)$ as $n \rightarrow \infty$; see [27, Lemma 2.6] or [28, Lemma 2.1].

(ii) Let $X$ be a Fréchet space and $T \in \mathscr{L}(X)$ be quasiMontel. Then $\tau_{s}-\lim _{n \rightarrow \infty}\left(T^{n} / n\right)=0$ if and only if $\tau_{b^{-}}$ $\lim _{n \rightarrow \infty}\left(T^{n} / n\right)=0$.

Again it suffices to show that $\tau_{s}-\lim _{n \rightarrow \infty}\left(T^{n} / n\right)=0$ implies $\tau_{b}-\lim _{n \rightarrow \infty}\left(T^{n} / n\right)=0$.

Arguing as in Remark 26(ii), for every $p \in \Gamma_{X}$ there exist $q \in \Gamma_{X}$ and $M>0$ such that (55) holds. Fix $p \in \Gamma_{X}, B \in$ $\mathscr{B}(X)$, and $\varepsilon>0$. Choose $q$ and $M$ according to (55). Since $T$ is a quasi-Montel operator, there is $\left\{M_{n}\right\}_{n=1}^{\infty} \subseteq \mathscr{M}(X)$ with $\left(T^{n}-M_{n}\right) \rightarrow 0$ in $\mathscr{L}_{b}(X)$ as $n \rightarrow \infty$. So there exists $m \in \mathbb{N}$ such that

$$
\sup _{x \in B} q\left(\left(T^{m}-M_{m}\right) x\right)<\frac{\varepsilon}{4 M} .
$$

But, $M_{m} \in \mathscr{M}(X)$ and so $M_{m}(B)$ is a relatively compact subset of $X$. It follows that there exist $x_{1}, \ldots, x_{k} \in X$ such that

$$
M_{m}(B) \subseteq \bigcup_{i=1}^{k}\left(x_{i}+\frac{\varepsilon}{4 M} \mathscr{U}_{q}\right),
$$

where $\mathcal{U}_{q}:=\{x \in X: q(x) \leq 1\}$. From (66) and (67) it follows that

$$
\begin{aligned}
T^{m}(B) & \subseteq\left(T^{m}-M_{m}\right)(B)+M_{m}(B) \\
& \subseteq \frac{\varepsilon}{4 M} \mathcal{U}_{q}+\bigcup_{i=1}^{k}\left(x_{i}+\frac{\varepsilon}{4 M} \mathcal{u}_{q}\right) \\
& \subseteq \bigcup_{i=1}^{k}\left(x_{i}+\frac{\varepsilon}{2 M} \mathcal{u}_{q}\right) .
\end{aligned}
$$


Fix $x \in B$. By (68) there exist $i \in\{1, \ldots, k\}$ and $z \in \mathscr{U}_{q}$ such that $T^{m}(x)=x_{i}+(\varepsilon / 2 M) z$. Then, by (55), for every $n>m$ we have that

$$
\begin{aligned}
p\left(\frac{T^{n} x}{n}\right) & =p\left(\frac{T^{n-m}}{n} T^{m}(x)\right) \\
& \leq p\left(\frac{T^{n-m} x_{i}}{n}\right)+\frac{\varepsilon}{2 M} p\left(\frac{T^{n-m} z}{n}\right) \\
& \leq p\left(\frac{T^{n-m} x_{i}}{n-m}\right)+\frac{\varepsilon}{2} .
\end{aligned}
$$

But, $p\left(T^{n-m} x_{i} /(n-m)\right) \rightarrow 0$ as $n \rightarrow \infty$. So, there exists $n_{0} \in$ $\mathbb{N}$ (depending only on $\left.x_{i}\right)$ such that $p\left(T^{n} x / n\right)<\varepsilon$, for every $n \geq n_{0}$. Since $x$ is arbitrary and the set $\left\{x_{1}, \ldots, x_{k}\right\}$ is finite, we can conclude that $\sup _{x \in B} p\left(T^{n} x / n\right) \rightarrow 0$ for $n \rightarrow \infty$. By the arbitrariness of $B$ and $p$ we have $\tau_{b}-\lim _{n \rightarrow \infty}\left(T^{n} / n\right)=0$.

Proposition 32. Let $X$ be a prequojection Fréchet space and let $T \in \mathscr{L}(X)$ satisfy $\tau_{s}-\lim _{n \rightarrow \infty}\left(T^{n} / n\right)=0$. If $T$ is quasiprecompact, then there exists a sequence $\left\{K_{n}\right\}_{n=1}^{\infty} \subseteq \mathscr{K}(X)$ such that $\tau_{b}-\lim _{n \rightarrow \infty}\left(T^{n}-K_{n}\right)=0$. In particular, $T$ is quasi-Montel as $\mathscr{K}(X) \subseteq \mathscr{M}(X)$.

Proof. The completeness of $X$ ensures that every precompact subset of $X$ is also relatively compact. By Fact 2 the operator $T$ is uniformly mean ergodic and so $\tau_{b}-\lim _{n \rightarrow \infty}\left(T^{n} / n\right)=0$. By [9, Theorems 1,2 and Satz 10] there exist $R \in \mathscr{L}(X)$ and a projection $P \in \mathscr{L}(X)$ commuting with $T$ such that $\operatorname{dim} P(X)<\infty$ and satisfying

$$
\begin{gathered}
T^{n}=R^{n}+T^{n} P, \quad n \in \mathbb{N}, \\
\mathbb{C} \backslash \mathbb{D} \subseteq \rho(R) .
\end{gathered}
$$

Since $P \in \mathscr{K}(X)$, also $K_{n}:=T^{n} P \in \mathscr{K}(X)$ for each $n \in \mathbb{N}$. Moreover, (70) yields $R^{n}=T^{n}(I-P)=(I-P) T^{n}$, for $n \in$ $\mathbb{N}$, and so $\tau_{b}-\lim _{n \rightarrow \infty} R^{n} / n=0$. Since (71) is equivalent to $\sigma(R) \subseteq \mathbb{D}$, it then follows from Theorem 10 applied to $R$ that $\tau_{b}-\lim _{n \rightarrow \infty} R^{n}=0$. It is then clear $($ see $(70))$ that $\left(T^{n}-K_{n}\right)=$ $R^{n} \rightarrow 0$ in $\mathscr{L}_{b}(X)$ as $n \rightarrow \infty$.

Remark 33. There exist quasi-Montel operators, even in quojection Fréchet spaces, which fail to be quasi-precompact.

(i) For $X:=\omega=\mathbb{C}^{\mathbb{N}}$, define the projection $P \in \mathscr{L}(X)$ via

$$
P x:=\left(x_{1}, 0, x_{3}, 0, x_{5}, \ldots\right), \quad x=\left(x_{n}\right)_{n} \in X .
$$

Since $X$ is a Montel space, all of its bounded subsets are relatively compact. It is then clear that $P \in \mathscr{M}(X)$, and hence, $P$ is surely quasi-Montel. Of course, $P \notin \mathscr{K}(X)$. On the other hand, since $\operatorname{Ker}(I-P)$ is infinite-dimensional, $P$ cannot be quasi-precompact [9, Satz 3].

(ii) Let $X$ be as in (i) and define the diagonal operator $T \in \mathscr{L}(X)$ by

$$
T x:=\left(x_{1}, \frac{1}{2} x_{2}, \frac{1}{3} x_{3}, \ldots\right), \quad x=\left(x_{n}\right)_{n} \in X .
$$

The same argument as in (i) shows that $T \in \mathscr{M}(X)$. In this case, in contrast to (i), the space $\operatorname{Ker}(I-T)=$ $\overline{\operatorname{span}}\{(1,0,0, \ldots)\}$ is finite-dimensional. However, $T$ still fails to be quasi-precompact $[9$, p. 24$]$.
Remark 34. The converse of Proposition 32 is not valid. Indeed, let $X:=\omega$ and let $T \in \mathscr{L}(X)$ be as Remark 33(ii), in which case $X$ is a quojection Fréchet space. For each $n \in \mathbb{N}$, let $K_{n} \in \mathscr{L}(X)$ be the finite rank operator given by

$$
K_{n} x:=\left(x_{1}, \frac{x_{2}}{2^{n}}, \frac{x_{3}}{3^{n}}, \ldots, \frac{x_{n}}{n^{n}}, 0,0, \ldots\right), \quad x=\left(x_{j}\right)_{j} \in X .
$$

Then $\mathscr{U}_{n}:=\left\{x \in X: \max _{1 \leq j \leq n}\left|x_{j}\right| \leq 1\right\}$ is a 0 -neighbourhood in $X$. Since $K_{n}$ has finite-dimensional range, it follows that $K_{n}\left(\mathcal{U}_{n}\right)$ is a relatively compact subset of $X$; that is, $K_{n} \in \mathscr{K}(X)$ for each $n \in \mathbb{N}$. Direct calculations show that the sequence of operators

$$
\begin{array}{r}
\left(T^{n}-K_{n}\right) x=\left(0, \ldots, 0, \frac{x_{n+1}}{(n+1)^{n}}, \frac{x_{n+2}}{(n+2)^{n}}, \ldots\right), \\
x=\left(x_{j}\right)_{j} \in X,
\end{array}
$$

converges to 0 in $\mathscr{L}_{s}(X)$ as $n \rightarrow \infty$. Since $X$ is a Montel space, also $\tau_{b}-\lim _{n \rightarrow \infty}\left(T^{n}-K_{n}\right)=0$. However, as noted in Remark 33(ii), the diagonal operator $T$ is not quasi-compact.

In view of Remark 33 the following result is an extension of Fact 2 above for prequojection Fréchet spaces (without the condition $\operatorname{dim} \operatorname{Ker}(I-T)<\infty)$.

Theorem 35. Let $X$ be a prequojection Fréchet space and $T \in \mathscr{L}(X)$. If $T$ is a quasi-Montel operator and $\tau_{s}$ $\lim _{n \rightarrow \infty}\left(T^{n} / n\right)=0$, then $T$ is uniformly mean ergodic.

Proof. We have the following two cases.

Case (I) ( $X$ is a quojection). The assumption $\tau_{s^{-}}$ $\lim _{n \rightarrow \infty}\left(T^{n} / n\right)=0$ ensures that we can proceed as in the proof of Proposition 8 to obtain $X=\operatorname{proj}_{j}\left(X_{j}, Q_{j, j+1}\right)$ in such a way that, for every $j \in \mathbb{N}$, there exists $T_{j}$ in $\mathscr{L}\left(X_{j}\right)$ satisfying $Q_{j} T=T_{j} Q_{j}$. Then also $Q_{j} T^{n}=T_{j}^{n} Q_{j}$ and $Q_{j}\left(T^{n} / n\right)=$ $\left(T_{j}^{n} / n\right) Q_{j}$, for every $j, n \in \mathbb{N}$. So, Lemma 6 (with $S_{n}:=T^{n} / n$, for $n \in \mathbb{N}$ ) implies that $\tau_{s}-\lim _{n \rightarrow \infty}\left(T_{j}^{n} / n\right)=0$ for all $j \in \mathbb{N}$.

Since $T$ is quasi-Montel, there exists a sequence $\left\{M_{n}\right\}_{n \in \mathbb{N}} \subseteq \mathscr{M}(X)$ such that $\tau_{b}-\lim _{n \rightarrow \infty}\left(T^{n}-M_{n}\right)=0$. From this it follows that the operator $T_{j}$, for any fixed $j \in \mathbb{N}$, is quasi-precompact. To see this, let $\widetilde{q}_{j}$ denote the norm of $X_{j}$ and $\varepsilon>0$. Since $p_{j}:=\widetilde{q}_{j} \circ Q_{j} \in \Gamma_{X}$, there exists $n \in \mathbb{N}$ such that

$$
\sup _{x \in B} p_{j}\left(T^{n} x-M_{n} x\right)<\frac{\varepsilon}{2},
$$

with $B \in \mathscr{B}(X)$ chosen such that $\widehat{B}_{j} \subseteq Q_{j}(B)$. Since

$$
\begin{aligned}
\sup _{x \in B} p_{j}\left(T^{n} x-M_{n} x\right) & =\sup _{x \in B} \widetilde{q}_{j}\left(Q_{j}\left(T^{n} x-M_{n} x\right)\right) \\
& =\sup _{x \in B} \widetilde{q}_{j}\left(T_{j}^{n} Q_{j} x-Q_{j} M_{n} x\right),
\end{aligned}
$$

it follows that

$$
T_{j}^{n}\left(\widehat{B}_{j}\right) \subseteq T_{j}^{n}\left(Q_{j}(B)\right) \subseteq Q_{j}\left(M_{n}(B)\right)+\frac{\varepsilon}{2} \widehat{B}_{j}
$$


Hence, by the relative compactness (hence, precompactness) of $Q_{j}\left(M_{n}(B)\right)$ in $X_{j}$, due to $M_{n} \in \mathscr{M}(X)$ and the continuity of $Q_{j}$, there exist $\widehat{x}_{1}, \ldots, \widehat{x}_{k} \in X_{j}$ such that

$$
T_{j}^{n}\left(\widehat{B}_{j}\right) \subseteq \bigcup_{i=1}^{k}\left(\widehat{x}_{i}+\varepsilon \widehat{B}_{j}\right) .
$$

By the arbitrariness of $\varepsilon>0$ it follows that $T_{j} \in \mathscr{L}\left(X_{j}\right)$ is quasi-precompact. As $X_{j}$ is a Banach space, $T_{j}$ is quasicompact [9, Theorem 3] and satisfies $T_{j}^{n} / n \rightarrow 0$ in $\mathscr{L}_{s}\left(X_{j}\right)$ for $n \rightarrow \infty$. By Fact 2, each operator $T_{j}$, for $j \in \mathbb{N}$, is uniformly mean ergodic. Then Lemma 7 implies that $T$ is also uniformly mean ergodic.

Case (II) ( $X$ is a prequojection). The condition $\tau_{s}-\lim _{n \rightarrow \infty}\left(T^{n} / n\right)=0$ actually means that $\tau_{b^{-}}$ $\lim _{n \rightarrow \infty}\left(T^{n} / n\right)=0$ because $T$ is quasi-Montel (see Remark 31(ii)). So, arguing as for Case (II) in the proof of Theorem 27, it follows that also $\tau_{b}-\lim _{n \rightarrow \infty}\left(\left(T^{\prime \prime}\right)^{n} / n\right)=0$. Moreover, by Remark 31(i) the operator $T^{\prime \prime}$ is quasi-Montel. Since $X^{\prime \prime}$ is a quojection Fréchet space, we can apply Case (I) to conclude that $T^{\prime \prime}$ is uniformly mean ergodic. Then $T$ is also uniformly mean ergodic as $\left.T^{\prime \prime}\right|_{X}=T$ with $X$ a closed subspace of $X^{\prime \prime}$.

Since the only Fréchet-Montel spaces which are normable are the finite-dimensional ones, the following result may be viewed as an analogue of the fact that $\operatorname{Ker}(\lambda I-T)$ is finite dimensional whenever $T$ is quasi-precompact; see Definition 3 and Theorem 1 of [9].

Proposition 36. Let $X$ be a Fréchet space and let $T \in \mathscr{L}(X)$ be a quasi-Montel operator. Then $\operatorname{Ker}(\lambda I-T)$ is a Fréchet-Montel space, for every $\lambda \in \mathbb{T}$.

Proof. It suffices to show that $\operatorname{Fix}(T)=\operatorname{Ker}(I-T)$ is a FréchetMontel space. Indeed, for every $\lambda \in \mathbb{T}$, the operator $\lambda^{-1} T$ is quasi-Montel if and only if $T$ is quasi-Montel, with $\operatorname{Ker}(\lambda I-$ $T)=\operatorname{Fix}\left(\lambda^{-1} T\right)$.

Let $\left\{r_{j}\right\}_{j=1}^{\infty}$ be any fundamental, increasing sequence of seminorms generating the lc-topology of $X$. Let $\left\{x_{k}\right\}_{k=1}^{\infty} \subseteq$ $\operatorname{Fix}(T)$ be a bounded sequence. Since $T$ is quasi-Montel, there exists $\left\{M_{n}\right\}_{n=1}^{\infty} \subseteq \mathscr{M}(X)$ such that $\tau_{b}-\lim _{n \rightarrow \infty}\left(T^{n}-M_{n}\right)=0$ and so, for every $j \in \mathbb{N}$, we have $\sup _{k \in \mathbb{N}} r_{j}\left(x_{k}-M_{n} x_{k}\right) \rightarrow 0$ as $n \rightarrow \infty$.

Since $\left\{x_{k}\right\}_{k=1}^{\infty}$ is bounded and each operator $M_{n}$, for $n \in \mathbb{N}$, is Montel, we may construct recursively subsequences $\left\{x_{k}^{n}\right\}_{k=1}^{\infty}$ of $\left\{x_{k}\right\}_{k=1}^{\infty}$ such that each $\left\{x_{k}^{n+1}\right\}_{k=1}^{\infty}$ is a subsequence of $\left\{x_{k}^{n}\right\}_{k=1}^{\infty}$ and $\left\{M_{n} x_{k}^{n}\right\}_{k=1}^{\infty}$ converges in $X$ for all $n \in \mathbb{N}$. Consider the diagonal sequence $\left\{x_{k}^{k}\right\}_{k=1}^{\infty}$. Clearly, $\left\{M_{n} x_{k}^{k}\right\}_{k=1}^{\infty}$ converges in $X$ for each $n \in \mathbb{N}$ (by observing that $\left\{M_{n} x_{k}^{k}\right\}_{k=1}^{\infty} \subseteq\left\{M_{n} x_{k}^{n}\right\}_{k \geq n}^{\infty}$ ). Fix $\varepsilon>0$ and $j \in \mathbb{N}$. Then, for every $k, k^{\prime} \in \mathbb{N}$ and $n \in \mathbb{N}$, we have

$$
\begin{aligned}
r_{j}\left(x_{k}^{k}-x_{k^{\prime}}^{k^{\prime}}\right) \leq & r_{j}\left(x_{k}^{k}-M_{n} x_{k}^{k}\right) \\
& +r_{j}\left(M_{n} x_{k}^{k}-M_{n} x_{k^{\prime}}^{k^{\prime}}\right)+r_{j}\left(M_{n} x_{k^{\prime}}^{k^{\prime}}-x_{k^{\prime}}^{k^{\prime}}\right)
\end{aligned}
$$

$$
\begin{aligned}
& \leq 2 \sup _{h \in \mathbb{N}} r_{j}\left(x_{h}-M_{n} x_{h}\right) \\
& +r_{j}\left(M_{n} x_{k}^{k}-M_{n} x_{k^{\prime}}^{k^{\prime}}\right),
\end{aligned}
$$

with $\sup _{h \in \mathbb{N}} r_{j}\left(x_{h}-M_{n} x_{h}\right) \rightarrow 0$ as $n \rightarrow \infty$. So, there is $n_{0} \in \mathbb{N}$ such that $\sup _{h \in \mathbb{N}} r_{j}\left(x_{h}-M_{n} x_{h}\right)<\varepsilon / 4$ for every $n \geq n_{0}$. But, $\left\{M_{n_{0}} x_{k}^{k}\right\}_{k=1}^{\infty}$ converges in $X$ and, hence, there is also $k_{0} \epsilon$ $\mathbb{N}$ such that $r_{j}\left(M_{n_{0}} x_{k}^{k}-M_{n_{0}} x_{k^{\prime}}^{k^{\prime}}\right)<\varepsilon / 2$ for all $k, k^{\prime} \geq k_{0}$. It follows that $r_{j}\left(x_{k}^{k}-x_{k^{\prime}}^{k^{\prime}}\right)<\varepsilon$ whenever $k, k^{\prime} \geq k_{0}$. By the arbitrariness of $j \in \mathbb{N}$ and $\varepsilon>0$ this means that $\left\{x_{k}^{k}\right\}_{k=1}^{\infty}$ is a Cauchy sequence in $X$ and so it converges in $X$. Since $X$ is a Fréchet space, this shows that $\operatorname{Fix}(T)$ is a Fréchet-Montel space.

Proposition 37. Let $X$ be a prequojection Fréchet space and $T \in \mathscr{L}(X)$ be a quasi-Montel operator. If $\tau_{s}-\lim _{n \rightarrow \infty}\left(T^{n} / n\right)=$ 0 , then $(I-T)(X)$ is closed.

Proof. By Theorem 35 the operator $T$ is uniformly mean ergodic. Also $\tau_{b}-\lim _{n \rightarrow \infty}\left(T^{n} / n\right)=0$. By [6, Theorem 3.5] this is equivalent to $(I-T)(X)$ being closed in $X$.

\section{Conflict of Interests}

The authors declare that there is no conflict of interests regarding the publication of this paper.

\section{Acknowledgments}

The research of the first two authors was partially supported by the projects MTM2010-15200 and GVA Prometeo II/2013/013 (Spain). The second author gratefully acknowledges the support of the Alexander von Humboldt Foundation.

\section{References}

[1] N. Dunford and J. T. Schwartz, Linear Operators I: General Theory, John Wiley \& Sons, New York, NY, USA, 1964.

[2] J. J. Koliha, "Power convergence and pseudoinverses of operators in Banach spaces," Journal of Mathematical Analysis and Applications, vol. 48, pp. 446-469, 1974.

[3] M. Mbekhta and J. Zemánek, "Sur le théorème ergodique uniforme et le spectre," Comptes Rendus de l'Académie des Sciences Paris, vol. 317, no. 12, pp. 1155-1158, 1993.

[4] U. Krengel, Ergodic Theorems, vol. 6, Walter de Gruyter, Berlin, Germany, 1985.

[5] M. Lin, "On the uniform ergodic theorem," Proceedings of the American Mathematical Society, vol. 43, pp. 337-340, 1974.

[6] A. A. Albanese, J. Bonet, and W. J. Ricker, "Convergence of arithmetic means of operators in Fréchet spaces," Journal of Mathematical Analysis and Applications, vol. 401, no. 1, pp. 160-173, 2013.

[7] Y. Katznelson and L. Tzafriri, "On power bounded operators," Journal of Functional Analysis, vol. 68, no. 3, pp. 313-328, 1986. 
[8] W. F. Eberlein, "Abstract ergodic theorems and weak almost peri odic functions," Transactions of the American Mathematical Society, vol. 67, pp. 217-240, 1949.

[9] V. A. Pietsch, "Quasi-präkompakte Endomorphismen und ein Ergodensatz in lokalkonvexen Vektorräumen," Journal für die Reine und Angewandte Mathematik, vol. 207, pp. 16-30, 1961.

[10] R. Meise and D. Vogt, Introduction to Functional Analysis, vol. 2, Clarendon Press, Oxford, UK, 1997.

[11] G. Köthe, Topological Vector Spaces II, Springer, New York, NY, USA, 1979.

[12] S. F. Bellenot and E. Dubinsky, "Fréchet spaces with nuclear Köthe quotients," Transactions of the American Mathematical Society, vol. 273, no. 2, pp. 579-594, 1982.

[13] J. Bonet, M. Maestre, G. Metafune, V. B. Moscatelli, and D. Vogt, "Every quojection is the quotient of a countable product of Banach spaces," in Advances in the Theory of Fréchet Spaces, T. Terzioğlu, Ed., vol. 287 of NATO ASI, pp. 355-356, Kluwer Academic Publishers, Dordrecht, The Netherlands, 1989.

[14] V. B. Moscatelli, "Fréchet spaces without continuous norms and without bases," The Bulletin of the London Mathematical Society, vol. 12, no. 1, pp. 63-66, 1980.

[15] P. Domański, "Twisted Fréchet spaces of continuous functions," Results in Mathematics, vol. 23, no. 1-2, pp. 45-48, 1993.

[16] H. Jarchow, Locally Convex Spaces, B. G. Teubner, Stuttgart, Germany, 1981.

[17] S. Dierolf and D. N. Zarnadze, "A note on strictly regular Fréchet spaces," Archiv der Mathematik, vol. 42, no. 6, pp. 549-556, 1984.

[18] A. A. Albanese, J. Bonet, and W. J. Ricker, "On the continuous Cesàro operator in certain function spaces," 2013, submitted.

[19] S. Dierolf and V. B. Moscatelli, "A note on quojections," Functiones et Approximatio Commentarii Mathematici, vol. 17, pp. 131-138, 1987.

[20] S. Önal and T. Terzioğlu, "Unbounded linear operators and nuclear Köthe quotients," Archiv der Mathematik, vol. 54, no. 6, pp. 576-581, 1990.

[21] D. Vogt, "On two problems of Mityagin," Mathematische Nachrichten, vol. 141, pp. 13-25, 1989.

[22] E. Behrends, S. Dierolf, and P. Harmand, "On a problem of Bellenot and Dubinsky," Mathematische Annalen, vol. 275, no. 3, pp. 337-339, 1986.

[23] V. B. Moscatelli, "Strongly nonnorming subspaces and prequojections," Studia Mathematica, vol. 95, no. 3, pp. 249-254, 1990.

[24] G. Metafune and V. B. Moscatelli, "Quojection and prequojections," in Advances in the Theory of Fréchet Spaces, T. Terzioğlu, Ed., vol. 287 of NATO ASI, pp. 235-254, Kluwer Academic Publishers, Dordrecht, The Netherlands, 1989.

[25] G. Metafune and V. B. Moscatelli, "Prequojections and their duals," in Progress in Functional Analysis, K. D. Bierstedt, J. Bonet, J. Horváth, and M. Maestre, Eds., vol. 170, pp. 215-232, North-Holland, Amsterdam, The Netherlands, 1992.

[26] A. A. Albanese, "A Fréchet space of continuous functions which is a prequojection," Bulletin de la Société Royale des Sciences de Liège, vol. 60, no. 6, pp. 409-417, 1991.

[27] A. A. Albanese, J. Bonet, and W. J. Ricker, "Grothendieck spaces with the Dunford-Pettis property," Positivity, vol. 14, no. 1, pp. 145-164, 2010.

[28] A. A. Albanese, J. Bonet, and W. J. Ricker, "On mean ergodic operators," in Vector Measures, Integration and Related Topics, G. P. Curbera, G. Mockenhaupt, and W. J. Ricker, Eds., vol. 201 of Operator Theory: Advances and Applications, pp. 1-20, Birkhäuser, Basel, Switzerland, 2010.
[29] A. A. Albanese, J. Bonet, and W. J. Ricker, " $C_{0}$-semigroups and mean ergodic operators in a class of Fréchet spaces," Journal of Mathematical Analysis and Applications, vol. 365, no. 1, pp. 142$157,2010$.

[30] A. A. Albanese, J. Bonet, and W. J. Ricker, "Uniform mean ergodicity of $C_{0}$-semigroups in a class of Fréchet spaces," Functiones et Approximatio Commentarii Mathematici. In press.

[31] H. R. Dowson, Spectral Theory of Linear Operators, vol. 12, Academic Press, London, UK, 1978.

[32] J. Zemánek, "On the Gelfand-Hille theorems," in Functional Analysis and Operator Theory, vol. 30 of Banach Center Publications, pp. 369-385, Polish Academy of Sciences, Warsaw, Poland, 1994.

[33] S. Dierolf and P. Domański, "Factorization of Montel operators," Studia Mathematica, vol. 107, no. 1, pp. 15-32, 1993.

[34] J. C. Díaz and P. Domański, "Reflexive operators with domain in Köthe spaces," Manuscripta Mathematica, vol. 97, no. 2, pp. 189-204, 1998.

[35] A. Grothendieck, Topological Vector Spaces, Gordon and Breach, London, UK, 1975.

[36] R. E. Edwards, Functional Analysis, Reinhart and Winston, New York, NY, USA, 1965.

[37] K. Floret, Weakly Compact Sets, vol. 801 of Lecture Notes in Mathematics, Springer, Berlin, Germany, 1980.

[38] B. Cascales and J. Orihuela, "On compactness in locally convex spaces," Mathematische Zeitschrift, vol. 195, no. 3, pp. 365-381, 1987.

[39] A. A. Albanese, J. Bonet, and W. J. Ricker, "Mean ergodic operators in Fréchet spaces," Annales Academiae Scientiarum Fennicae, vol. 34, no. 2, pp. 401-436, 2009.

[40] M. Altman, "Mean ergodic theorem in locally convex linear topological spaces," Studia Mathematica, vol. 13, pp. 190-193, 1953.

[41] M. V. Deshpande and S. M. Padhye, "An ergodic theorem for quasi-compact operators in locally convex spaces," Journal of Mathematical and Physical Sciences, vol. 11, no. 2, pp. 95-104, 1977. 


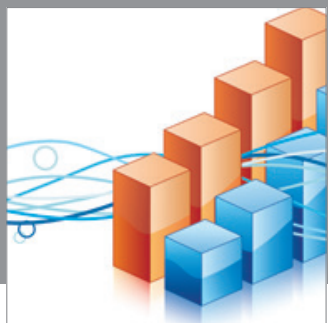

Advances in

Operations Research

mansans

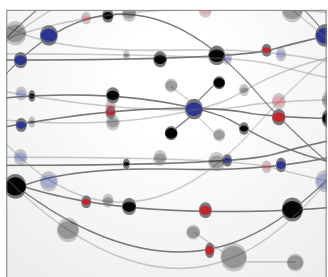

The Scientific World Journal
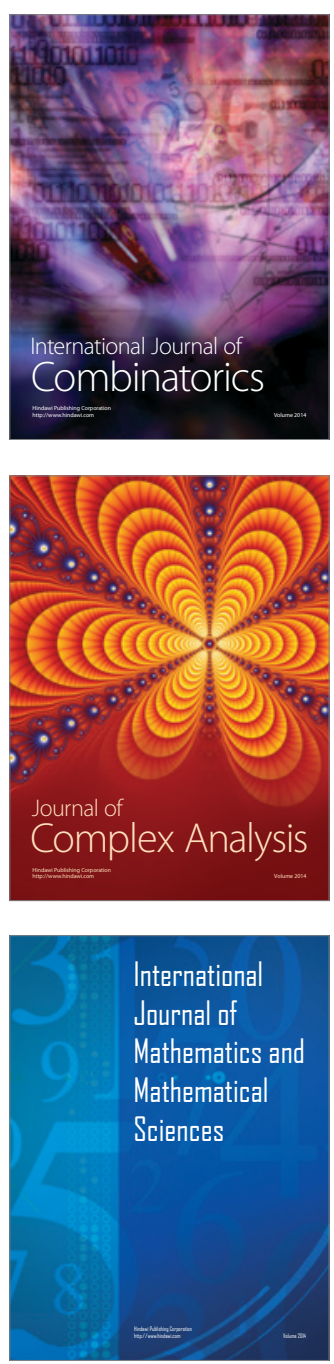
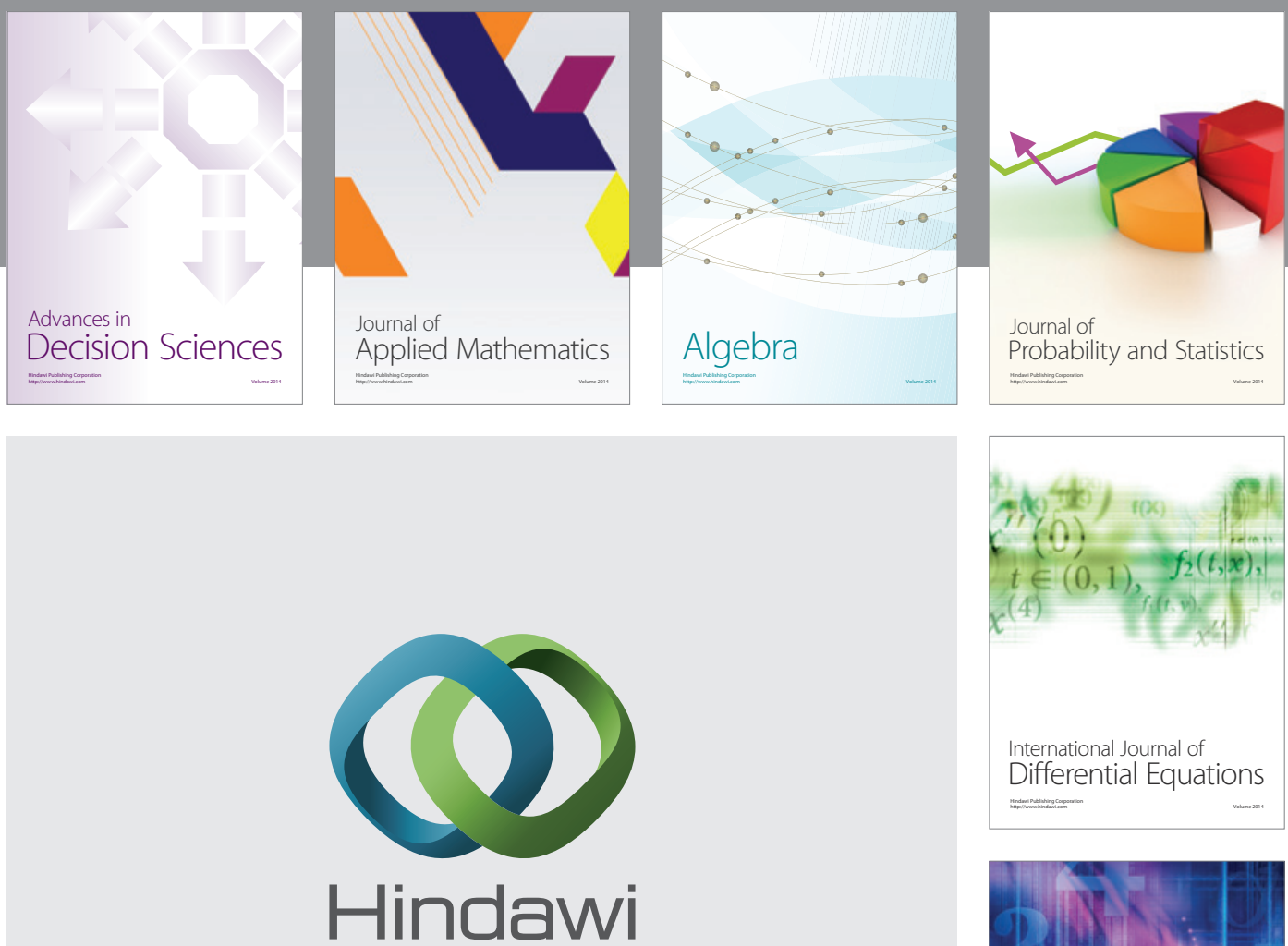

Submit your manuscripts at http://www.hindawi.com
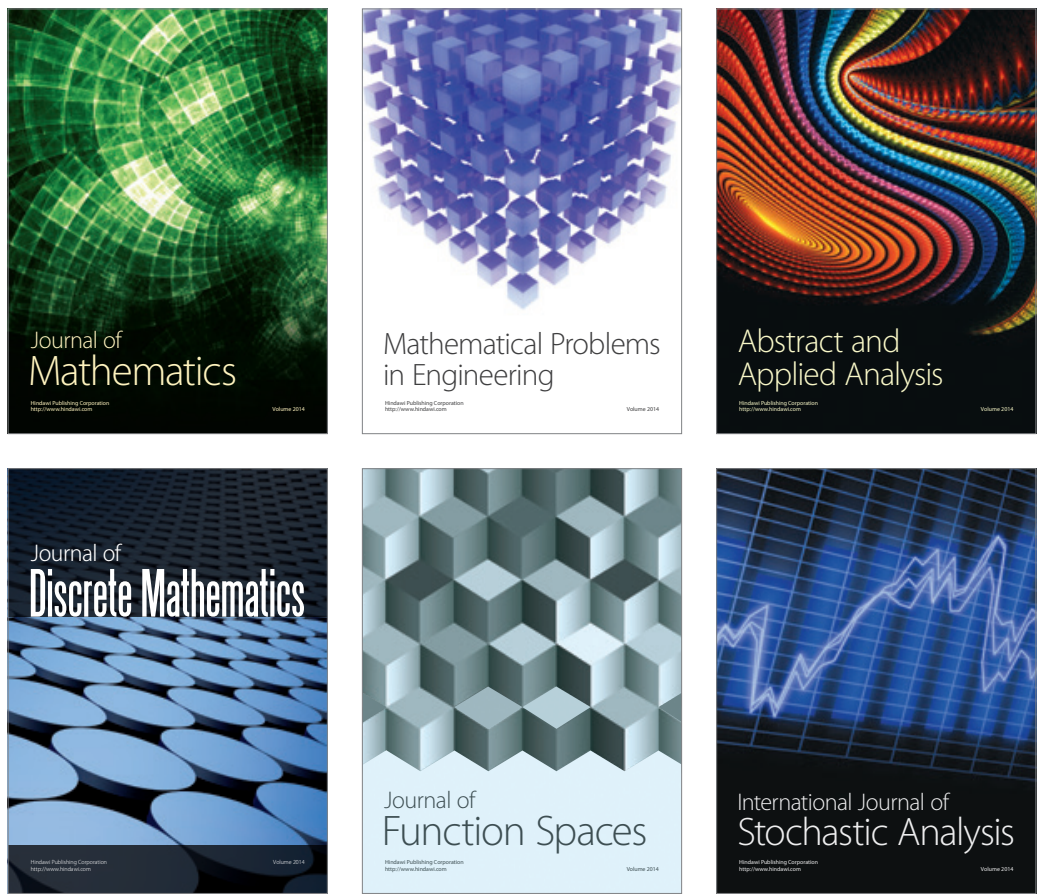

Journal of

Function Spaces

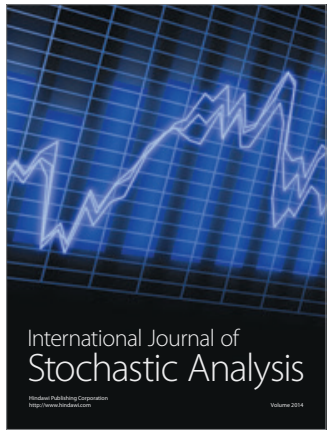

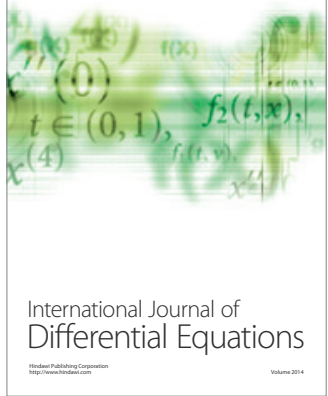
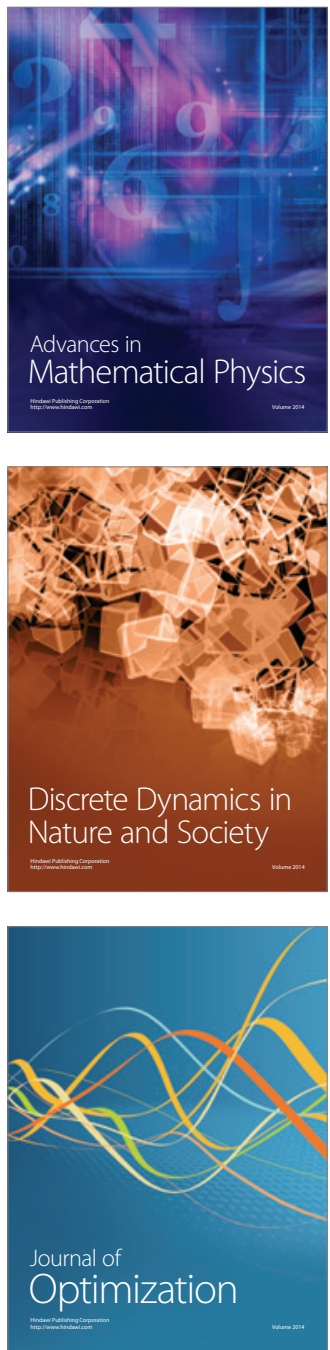